ben.swarts@cmich.edu.

Conflicts of interest

There are no conflicts to declare. 


\section{Overview of trehalose}

Over the past few decades, the non-mammalian disaccharide trehalose has emerged from relative obscurity to become an intensely studied sugar that commands the attention of scientists from diverse fields. Early research on trehalose focused on its natural occurrence and biological functions, which revealed that the disaccharide fulfills functions in energy storage and stress protection in microbes, plants, and invertebrates. ${ }^{1}$ These functions originate from the unique structure of trehalose, which consists of two glucose units joined by an a,a-1,1-glycosidic bond (Figure 1). Trehalose is $C_{2}$-symmetric and non-reducing, making it chemically stable relative to reducing sugars. On the other hand, trehalose can be readily enzymatically hydrolyzed into its constituent glucose monomers by trehalases, hence its utility as a biological source of carbon and energy. Trehalose is a remarkable biological stabilizer, as it protects macromolecules, cells, and tissues from desiccation, heat-shock, and other stresses. ${ }^{2}$ While trehalose's mechanism of protection remains a matter of debate, recent evidence supports the so-called preferential hydration model, wherein trehalose does not exert its stabilizing effects through direct interactions with a macromolecule, but rather through reducing the dynamics of the surrounding water layer and thus the macromolecule itself. $^{3}$ Regardless of the mechanism, trehalose's bioprotective properties have driven its adoption as an additive in a plethora of foods, pharmaceuticals, and other products. ${ }^{2}$ More recently, trehalose has garnered attention from the biomedical field because numerous reports describe its ability to alleviate disease burden in animal models of various cardiometabolic and neurodegenerative diseases. ${ }^{4,5}$ Trehalose's ubiquity as an additive, and its emergence as a potential therapeutic, were propelled by the introduction of an efficient industrial enzymatic process for its production in $1995,{ }^{6}$ and further bolstered by its "Generally Regarded as Safe" status, assigned by the U.S. Food and Drug Administration in 2000.

\section{Importance of trehalose to bacterial physiology and virulence}

Elucidating the roles of trehalose in bacterial growth and virulence is a critical area of biomedical research. Trehalose is not biosynthesized or required by humans, and thus its associated metabolic pathways in bacteria are potential targets for antibiotic and diagnostic development. Many types of bacteria, including numerous pathogens, are capable of synthesizing and/or degrading trehalose, although trehalose metabolism and its contributions to bacterial virulence vary immensely from organism to organism (Figures 2 and 3). Escherichia coli, the widely studied Gram-negative model organism, biosynthesizes trehalose through the cytoplasmic OtsAB pathway, which is the predominant route of trehalose synthesis in bacteria (Figure 2A). ${ }^{1,7}$ OtsA couples glucose-6-phosphate (Glc-6-P) and uridine diphosphate glucose (UDP-Glc) to form trehalose-6-phosphate (Tre-6-P), which is subsequently dephosphorylated by OtsB to give trehalose (also referred to as the Tre-6-P synthase/Tre-6-P phosphatase (TPS/TPP) pathway). ${ }^{7}$ E. coli can degrade trehalose through multiple routes, including via the cytosolic and periplasmic trehalases $\operatorname{TreF}^{8}$ and $\operatorname{Tre} A,{ }^{9}$ which hydrolyze trehalose to two glucose units, and the cytosolic phosphotrehalase TreC, ${ }^{10}$ which hydrolyzes Tre-6-P to glucose and Glc-6-P. Transport of trehalose in dual-membrane E. coli occurs in multiple ways, as trehalose can be exported across the plasma membrane (PM) into the periplasm, while periplasmic trehalose can be imported back into the 
cytoplasm via phosphoenolpyruvate-dependent phosphotransferase systems (PTS), either directly as Tre-6-P, or as Glc-6-P following cleavage into glucose by TreA. ${ }^{11}{ }^{12}$ Porins allow non-specific passage of small solutes, including trehalose, across the outer membrane (OM), thus E. coli and similar Gram-negative bacteria also possess machinery to take up and utilize exogenous trehalose. ${ }^{11}$ In E. coli and many other trehalose-synthesizing organisms, the disaccharide is classically known to be induced and promote survival in vitro in response to various stresses, such as osmotic shock, changes in temperature, desiccation, and nutrient deprivation. ${ }^{1}$ While the importance of trehalose biosynthesis to the virulence of fungal pathogens is well-established, ${ }^{13}$ its significance in the context of many mammalian bacterial infections remains unclear. On the other hand, trehalose degradation pathways have been implicated in the virulence of multiple bacterial pathogens, ${ }^{145}$ including intestinal pathogenic E. coli, ${ }^{14,}{ }^{15}$ Burkholderia pseudomallei, ${ }^{16,17}$ and Clostridioides difficile, ${ }^{18,}, 19$ the latter of which is discussed further below.

Some bacteria do not biosynthesize their own trehalose, but can acquire it from their environment and utilize it as a carbon source via degradative pathways to promote growth. As representative examples, the Gram-positive organisms Bacillus subtilis and Clostridioides difficile (formerly Clostridium difficile) share a pathway by which exogenous trehalose can be imported via a PTS to generate cytoplasmic Tre-6-P, which is subsequently degraded to glucose and Glc-6-P via the phosphotrehalase TreA (Figure 2B). ${ }^{19-22}$ This pathway, which is analogous to the PTS-TreC system in E. coli (Figure 2A), permits growth on externally supplied trehalose. In 2018, it was reported that $C$. difficile, which causes opportunistic intestinal infections in humans that are extremely challenging to treat, has evolved distinct mechanisms that enable highly efficient utilization of trehalose. ${ }^{18,} 19$ Expression of the $C$. difficile phosphotrehalase TreA is under the control of the Tre-6-Pbinding trehalose repressor, TreR. The epidemic $C$. difficile strains RT027 and RT017 have single point mutations in $t r e R$ that confer $>500$-fold sensitivity to trehalose, which enabled these strains to grow on much lower trehalose concentrations than typical strains. ${ }^{18,} 19$ Interestingly, a different epidemic strain, $C$. difficile RT078, can also grow on low trehalose due to the acquisition of an additional cluster of trehalose metabolism genes, namely a second PTS that mediates high-efficiency trehalose uptake from the environment. ${ }^{18,19}$ Both of these adapted trehalose utilization mechanisms were shown to provide in vitro growth advantages to $C$. difficile, which led to the hypothesis that escalating consumption of trehalose by humans may have contributed to the spread and virulence of these epidemic $C$. difficile strains over the past two decades. ${ }^{18,19,23}$ However, the available evidence from mouse infection models ${ }^{18,} 19,24$ is mixed and, along with human epidemiology data, ${ }^{25,} 26$ does not unequivocally support this hypothesis. Whereas some reports ${ }^{18,19}$ suggested that trehalose enhanced RT027 disease severity via toxin elevation, these data are counterbalanced by mouse infection models showing that trehalose did not increase RT027 disease severity, bacterial burden, toxin level, or colorectal inflammation. ${ }^{24}$ Further investigation is needed to assess the potential contributions of trehalose metabolism to $C$. difficile infection and, correspondingly, its potential as a therapeutic target. In the meantime, these studies illustrate the diversity of trehalose metabolic pathways that occur and evolve in bacteria, even between different strains of the same species, and also call attention to the possibility that trehalose consumption can impact the trehalose-metabolizing population of 
the human microbiota. Indeed, numerous other genera that include human commensal or pathogenic bacteria have been experimentally demonstrated to have trehalose synthesis and/or degradation capabilities, ${ }^{27-35}$ although in most cases these pathways are not wellcharacterized and their roles in host interactions or infection are not known.

Perhaps the most interesting and widely appreciated trehalose-utilizing microbes are those in Mycobacterium and related genera (Figure 3), which include globally important human pathogens such as those that cause tuberculosis (TB), leprosy, diphtheria, and opportunistic non-tuberculous mycobacterial (NTM) infections. TB is an urgent global health problem, as there were an estimated 10 million cases and 1.2 million deaths in 2019, and emerging drugresistance continues to stress TB control programs. ${ }^{36}$ Mycobacteria are distinguished from Gram-negative and Gram-positive bacteria by their lipid-rich, dual-membrane cell envelope, which is typified by carbohydrate-linked mycolic acids that form the uniquely protective mycobacterial outer membrane, or mycomembrane. ${ }^{37,}{ }^{38}$ As with $E$. coli, the major route of trehalose biosynthesis in mycobacteria is the OtsAB pathway, ${ }^{39-41}$ and trehalose can be broken down into two glucose units by a trehalase located in the cytoplasm. ${ }^{42}$ In part through the TreS and $\operatorname{Tre}(\mathrm{X}) \mathrm{YZ}$ pathways, cytoplasmic trehalose can also be cycled into and out of (i) intracellular polysaccharides, which function in energy storage, or (ii) capsular polysaccharides, which appear to contribute to immune evasion during mycobacterial infection. ${ }^{43,44}$ Most importantly, trehalose is the central mediator of mycomembrane biosynthesis due to its mycolic acid shuttling function. In the cytoplasm, trehalose is converted to trehalose monomycolate (TMM) ${ }^{45}$ which is then exported to the periplasm by the flippase MmpL3. ${ }^{46,47}$ Once beyond the plasma membrane, TMM serves as a mycoloyl donor, as its acyl chain is transferred to various acceptor molecules by $\mathrm{Ag} 85$ mycoloyltransferases. ${ }^{48-50}$ This process generates the major mycolic acid-containing components of the mycomembrane, including inner-leaflet arabinogalactan-linked mycolates (AGM), outer-leaflet trehalose dimycolate (TDM), related species like glucose and glycerol monomycolate, and, in some organisms, $O$-mycoloylated proteins. ${ }^{37}$ In the form of TDM, trehalose is a major structural and immunoactive component of the mycobacterial envelope. 51, 52 TDM can be broken down to TMM and free mycolic acid by Tdmh, a mycomembraneremodeling mechanism that facilitates the balance of nutrient acquisition and defense during stress. ${ }^{53,54}$ In addition to TDM, mycobacteria produce numerous other trehalose-based glycolipids, including diacyltrehalose, polyacyltrehalose, trehalose polyphleates, and trehalose-containing lipooligosaccharides and sulfolipids. ${ }^{55-58}$ Trehalose transport across the mycomembrane is likely porin-mediated, similar to $E$. coli, whereas uptake through the $\mathrm{PM}$ in mycobacteria proceeds via the trehalose-specific $\mathrm{ABC}$ transporter LpqY-SugABC, 59,60 which is in contrast to the trehalose PTSs found in many species, such as E. coli, $B$. subtilis, and C. difficile. LpqY-SugABC recycles free trehalose released via Ag85 mycoloyltransferase activity, and it can also mediate uptake of exogenous trehalose from the environment. ${ }^{59,60}$ Collectively, this remarkable network of trehalose metabolic pathways is extremely important to mycobacteria, with many steps being essential for viability (e.g., trehalose biosynthesis, MmpL3, Ag85), and other steps contributing to mycobacterial stress adaptation and/or virulence (e.g., LpqY-SugABC, TreS, Tdmh). Thus, there has been significant effort to investigate these mycobacterial pathways and target them for diagnostic and therapeutic applications, as discussed extensively in this article. ${ }^{61-63}$ Surprisingly, in a 
recent study TDM-like trehalose phospholipids were discovered in Salmonella species and some strains of $E$. coli, ${ }^{64}$ suggesting that trehalose glycolipids and their associated metabolic pathways may be more widespread than originally thought.

\section{Synthetic trehalose analogues as tools for investigating and targeting bacterial trehalose metabolism}

The increasingly recognized significance of trehalose to diverse bacteria, including major pathogens such as $M$. tuberculosis and $C$. difficile, combined with the sugar's absence in humans, motivate further investigation of bacterial trehalose metabolic pathways. New tools need to be developed and applied to better understand the contributions of trehalose metabolism to bacterial virulence, which in turn may lead to improvements in the detection and treatment of bacterial infections. As illustrated in Figures 2 and 3, trehalose-utilizing bacteria possess mechanisms for the uptake of exogenous trehalose, which presents opportunities for the rational design and development of synthetic trehalose-based imaging probes or inhibitors that can potentially report on or block bacterial trehalose metabolism, respectively. Indeed, over the past several years, trehalose analogues have emerged as valuable research tools, predominantly in the field of mycobacteria research. The first examples of trehalose-based imaging probes included fluorescein isothiocyanate-modified keto trehalose (FITC-Tre) and azide-modified "clickable" trehalose (TreAz) analogues that hijack mycobacterial trehalose metabolism to incorporate the label and allow live-cell imaging of trehalose glycolipids (Figure 4) ${ }^{65}, 66$ Metabolically incorporated azido sugars, including TreAz analogues, are detectable following bioorthogonal click chemistry reaction with complementary terminal alkyne- or cyclooctyne-modified fluorophore reagents. ${ }^{67} \mathrm{In}$ addition, because trehalose metabolism is essential for viability in mycobacteria, several early studies also investigated synthetic trehalose analogues as potential inhibitors of mycobacterial growth, with some compounds exhibiting growth reduction in the 100-1000 $\mu \mathrm{M}$ range (e.g., 6-TreAz, Figure 4). ${ }^{49}, 65,68$ These studies provided proof-of-concept that synthetic trehalose analogues can serve as tools to study mycobacterial trehalose metabolism, and that they may have potential for the diagnosis and treatment of mycobacterial infections.

Despite the potential of trehalose analogues as tools for advancing mycobacteria research and possibly contributing to infection control, these efforts are still in their infancy. Moreover, until recently the application of trehalose analogues to non-mycobacterial species has been virtually non-existent. A major hurdle to realizing the potential of trehalose analogues is the lack of practical and efficient synthetic methods (Figure 5). Trehalose's a,a-1,1-glycosidic bond and $C_{2}$ symmetry make analogue synthesis via traditional chemical approaches challenging due to the requirement of either: (i) laborious trehalose desymmetrization and regioselective hydroxyl manipulations requiring extensive protecting group chemistry (Scheme 1A); or (ii) chemical glycosylation to form the a,a-1,1-glycosidic bond, which is notoriously difficult to achieve with good stereoselectivity and also requires protecting groups (Scheme 1B). As previously reviewed, various specialized chemical synthesis methods in these categories have been developed to access diverse trehalose derivatives, ${ }^{69,70}$ including complex trehalose glycolipids. ${ }^{71}$ However, even for synthesizing 
relatively simple probes and inhibitors such as those shown in Figure 4, these approaches are lengthy (typically $\sim 5-10$ steps), inefficient (often $<10 \%$ overall yield), and demand high synthetic expertise to execute. As an alternative to chemical synthesis, our group and others have turned to the creation of chemoenzymatic synthesis methods, which in principle allow the use of trehalose-synthesizing enzymes to stereo- and regioselectively couple simple, unprotected substrates to efficiently and conveniently produce trehalose analogues while generating minimal hazardous waste (Scheme 1C). Previously, the Desmet group reviewed the enzymatic synthesis of hydrolytically stable trehalose analogues for food industry applications, ${ }^{72}$ and our group reviewed, from a broader perspective, the development and applications of trehalose analogues. ${ }^{73}$ In this Feature article, we provide a contextual account of our team's development of a chemoenzymatic method for trehalose analogue synthesis employing a thermostable trehalose synthase enzyme, TreT from Thermoproteus tenax. Then, in three vignettes, we highlight how the TreT method has accelerated the development of trehalose-based imaging probes and inhibitors that target trehalose-utilizing bacterial pathogens.

\section{The use of enzymes in trehalose and trehalose analogue synthesis}

Microbial trehalose biosynthesis and degradation pathways, including those shown in Figures 2 and 3, involve numerous enzymes that can, in principle, be exploited to develop chemoenzymatic methods for trehalose analogue synthesis. Notably, the industrial-scale process for production of native trehalose, developed by Hayashibara Co., is based on an enzymatic system that converts starch-derived maltopentaose to trehalose (Scheme 2). ${ }^{74}$ The key steps of this process are (i) isomerization of the maltopentaose reducing end glycosidic bond from an a-1,4- to an a,a-1,1-linkage, catalyzed by TreY (also referred to as maltooligosyl trehalose synthase (MTSase)), followed by (ii) hydrolysis of the intermediate a-maltotriosyl trehalose to release trehalose, catalyzed by TreZ (also referred to as maltooligosyl trehalose trehalohydrolase (MTHase)). ${ }^{6}$ Analogous pathways are present in numerous organisms, including mycobacteria ( $\operatorname{Tre}(\mathrm{X}) \mathrm{YZ}$ pathway, Figure 3), although the industrial process utilizes enzymes from the soil bacterium Arthrobacter ramosus that are mesophilic and have good expression characteristics. ${ }^{75}$ While the Tre(X)YZ enzymatic synthesis process has been extraordinarily successful for the cost-effective production of native trehalose, ${ }^{2}$ it cannot be practically adapted to produce trehalose analogues, since it would require controlled site-selective modification of a complex carbohydrate starting material, and, subsequently, that the modified complex substrate be efficiently processed by multiple enzymes.

In contrast to the $\operatorname{Tre}(\mathrm{X}) \mathrm{YZ}$ system, trehalose metabolic pathways that operate on simpler substrates are more suitable for trehalose analogue synthesis. The major pathway for trehalose biosynthesis in nature, the OtsAB (TPS/TPP) pathway (Figures 2 and 3), is a reasonable option for analogue synthesis, as it utilizes the relatively simple monosaccharide derivatives Glc-6-P and UDP-Glc as substrates. In 2011, Barry, Davis, and co-workers reported an OtsAB-based synthesis of 2-deoxy-2-[ $\left.{ }^{19} \mathrm{~F}\right]$ fluoro-D-trehalose $\left({ }^{19} \mathrm{~F}-2\right.$-FDTre $),{ }^{65}$ which represented the first step toward the development of ${ }^{18} \mathrm{~F}$-modified trehalose as a potential positron emission tomography (PET) probe for in vivo imaging of mycobacterial infections (this application is discussed further below). The synthesis, shown in Scheme 3, 
highlighted the advantages and limitations of employing the OtsAB system for analogue synthesis. Because OtsA (TPS) utilizes Glc-6-P and UDP-Glc as substrates to produce Tre-6-P, 2-deoxy-2- $\left[{ }^{19} \mathrm{~F}\right]$ fluoro-D-glucose 6-phosphate $\left({ }^{19} \mathrm{~F}-2\right.$-FDG-6-P) was chosen as the substrate analogue. Since ${ }^{19}$ F-2-FDG-6-P is not commercially available in appreciable quantities, it was synthesized from 2-deoxy-2-[ $\left.{ }^{19} \mathrm{~F}\right]$ fluoro-D-glucose $\left({ }^{19} \mathrm{~F}-2-\mathrm{FDG}\right)$ and ATP under yeast hexokinase catalysis, using a procedure from Chenault et al. ${ }^{76}$ The key glycosylation proceeded smoothly, with $E$. coli OtsA catalyzing the coupling of ${ }^{19} \mathrm{~F}-2$ FDG-6-P and UDP-Glc to form ${ }^{19} \mathrm{~F}-2-\mathrm{FDTre}-6-\mathrm{P}$ in $63 \%$ yield and with complete a,a-1,1stereoselectivity. ${ }^{65}$ In the final step of the synthesis, ${ }^{19} \mathrm{~F}-2-\mathrm{FDTre}-6-\mathrm{P}$ was dephosphorylated by alkaline phosphatase in nearly quantitative yield to deliver the target molecule, ${ }^{19} \mathrm{~F}-2$ FDTre. ${ }^{65}$ Given that 2-position-modified trehalose analogues can take approximately 10 steps to chemically synthesize from trehalose using the desymmetrization approach (Scheme 1A), this synthesis exemplified the power of the chemoenzymatic approach. However, beyond this example and efforts to extend it to the radiosynthesis of the ${ }^{18} \mathrm{~F}$-modified version, ${ }^{77}$ the OtsAB (TPS/TPP) pathway has not been applied to the synthesis of other trehalose analogues. This is likely due to the need for multiple enzymatic transformations, which significantly decreases speed and efficiency, and potentially amplifies substrate tolerance problems.

The earliest and one of the more successful approaches to the chemoenzymatic synthesis of trehalose analogues interestingly employs trehalose phosphorylases (TrePs), a group of enzymes that appear to predominantly degrade trehalose in vivo. TrePs catalyze the reversible conversion of trehalose to glucose and Glc-1-P, with retaining TreP enzymes giving a-Glc-1-P and inverting TreP enzymes giving $\beta$-Glc-1-P (Scheme 4). ${ }^{78}$ Due to the high energy content of Glc-1-P, the TreP reaction can be reversed to generate trehalose. Thus, in principle, TrePs can generate trehalose analogues from $\mathrm{a}$ - or $\beta$-Glc-1-P donors and alternative monosaccharide acceptors in one step, so long as the requisite acceptor substrate tolerance exists. This is potentially a major advantage because there is a wide range of monosaccharides to replace glucose in the reaction that are commercially available and affordable. Desmet and co-workers reviewed TreP-catalyzed trehalose analogue synthesis in depth, ${ }^{72}$ so here we briefly describe some of the key benefits and limitations of these enzymes for synthesis, and some of the analogues made. While the retaining TrePs utilize a readily available and relatively inexpensive donor sugar, a-Glc-1-P, to date these enzymes have exhibited low substrate tolerance with respect to glucose, and their protein characteristics (e.g., expression yield and stability) are poor. ${ }^{72}$ Thus, there has been limited application of retaining TrePs in synthesis. By contrast, inverting TrePs, which are widely distributed in microbes, including in thermophilic organisms, have shown better substrate tolerance (Figure 5). ${ }^{72}$ The first TreP characterized, from the alga Euglena gracilis, had limited substrate tolerance, but was capable of producing 6-deoxy-D-trehalose (6-DeoxyTre) and xylotrehalose (XyloTre) from 6-deoxy-D-glucose and D-xylose, respectively, albeit in very low yields $(<1 \%) .{ }^{79,80}$ More recently, the thermostable inverting TrePs from the bacteria Thermoanaerobacter brockii and Caldanaerobacter subterraneus have shown activity for a broader range of alternative acceptor substrates (e.g., D-galactose, D-xylose, Larabinose, D-mannose, D-fucose, and L-fucose), but the activities relative to glucose were generally low, as even the best substrates exhibited $20-40 \%$ relative activity. ${ }^{72,81,82}$ There 
has been some success in enzyme engineering efforts to improve the activity of $T$. brockii TreP toward galactose and mannose. ${ }^{83}$ To date, however, only a few trehalose analogues have been produced by inverting TrePs on a scale large enough to permit product characterization (Figure 5). Indeed, a significant problem with inverting TrePs is that they must utilize $\beta$-Glc-1-P as the donor, which is currently either unavailable or extremely expensive, prohibiting large-scale TreP reactions. ${ }^{72}$ One way around this problem is to use TreP to generate $\beta$-Glc-1-P in situ from trehalose in the presence of an alternative acceptor, which has been done to convert galactose into the trehalose stereoisomer, lactotrehalose (LactoTre, Figure 5), although this approach generates a complex reaction mixture that requires laborious purification procedures. ${ }^{84}$ Thus, improved methods to produce pure $\beta$ Glc-1-P, which are in development ${ }^{85,} 86$ will greatly improve the inverting TreP catalysis approach to trehalose analogue synthesis.

While progress has been made using both the OtsAB and TreP pathways for trehalose analogue synthesis, there are clear drawbacks to these systems that motivate the development of alternative approaches. Over the past several years, our group has been exploring the synthetic utility of the TreT trehalose biosynthesis pathway, which is widely distributed in archaea but apparently occurs infrequently in bacteria. TreT is a $\mathrm{Mg}^{2+}$ dependent trehalose glycosyltransferring synthase that catalyzes the coupling of glucose and UDP-Glc to form trehalose (Scheme 5A). ${ }^{87}$ In principle, TreT should be able to produce trehalose analogues in a single step from a monosaccharide acceptor and the relatively inexpensive donor UDP-Glc. We were encouraged by some earlier reports demonstrating that TreT enzymes from the archaeal hyperthermophiles Pyrococcus horikoshii and Thermotoga maritima could accept galactose or mannose in lieu of glucose to generate the trehalose stereoisomers LactoTre and MannoTre, respectively (both structures are shown in Figure 6) ${ }^{88-90}$ However, we were most intrigued by the so-called unidirectional TreT pathway in the hyperthermophilic archaeon Thermoproteus tenax, which, in contrast to TreT pathways in other organisms, is active only in the direction of trehalose synthesis. ${ }^{91}$ Species in the Thermoproteus genus are hydrogen-sulfur autotrophs that primarily dwell in hot springs and water holes, growing at temperatures up to $95^{\circ} \mathrm{C}$. We hypothesized that, if the thermostable T. tenax TreT possessed good substrate tolerance, it would be an ideal candidate for the one-step chemoenzymatic synthesis of trehalose analogues.

\section{Development of the TreT chemoenzymatic synthesis method}

In 2013, we set out to investigate the potential of $T$. tenax TreT for trehalose analogue synthesis (Scheme 5B). We expressed His-tagged TreT in E. coli using an L-arabinoseinducible promoter, then lysed the cells and employed $\mathrm{Ni}^{2+}$ affinity chromatography to obtain the enzyme in a modest yield of $4 \mathrm{mg} / \mathrm{L} .{ }^{92}$ Using an HPLC assay to assess conversion of substrate to product, we screened TreT activity on a wide variety of commercially available and custom-synthesized monosaccharides in place of the native acceptor glucose. During this exploratory stage, reactions were performed on a small scale $(50 \mu \mathrm{L})$ and consisted of $10 \mathrm{mM}$ glucose surrogate, $40 \mathrm{mM}$ UDP-Glc donor, $\sim 10 \mu \mathrm{M}$ TreT, and $50 \mathrm{mM}$ HEPES buffer at $\mathrm{pH}$ 7.4. After confirming the thermostable enzyme was active at elevated temperatures, we ran the screening reactions for $1 \mathrm{~h}$ at $70{ }^{\circ} \mathrm{C}$ prior to quenching and HPLC analysis. We were encouraged to find that $T$. tenax TreT exhibited broad natural substrate 
tolerance, delivering diverse trehalose analogues in one step, and often with quantitative conversion. ${ }^{92}$ While specific analogues' applications are discussed below, we note that a variety of deoxy-, deoxyfluoro-, azidodeoxy-, deoxythio-, and stereochemically modified analogues were accessible (Figure 6). ${ }^{92}$ Generally, these compounds take 5-10 steps to produce via chemical synthesis, and the overall yields are often less than $\sim 5 \%$, thus the TreT method provides outstanding efficiency for these structures. The main exception in terms of substrate tolerance was 4-position-modified monosaccharides, which were not welltolerated, although the modest yields observed for some of these reactions indicated potential for improvement. In contrast to some of the reports on TreP analogue synthesis, we did not only measure enzyme activity in these assays, but directly observed the product by HPLC and confirmed its identity by high-resolution MS. ${ }^{92}$ Selected analogues were also produced on a semi-preparative scale $(\sim 10 \mathrm{mg})$ in high isolated yields and fully characterized by NMR spectroscopy, proving the formation of the a,a-1,1-glycosidic linkage. ${ }^{92}$ This preliminary work, which was published in $2014,{ }^{92}$ paved the way for continued development of the TreT chemoenzymatic synthesis method.

We next sought to optimize various aspects of the TreT method to improve its overall efficiency (Scheme 5B). ${ }^{93,94}$ To facilitate these efforts, we began to deploy a highthroughput, highly sensitive luminescence assay that detects UDP released during glycosyltransferase-catalyzed reactions. ${ }^{94}$ We more thoroughly probed the dependence of the TreT reaction on temperature, buffer, $\mathrm{pH}$, and salt concentration, finding that the enzyme retained optimum activity at $70-90{ }^{\circ} \mathrm{C}$, in a range of buffers near neutral $\mathrm{pH}(\sim 7-8)$, and at various salt concentrations. ${ }^{94}$ These findings confirmed that the reaction temperature could be modulated in an advantageously high range and that there was flexibility for other reaction variables as well. We also carefully investigated the acceptor:donor ratio and found that most reactions reached complete conversion at a 1:1.5 or 1:2 ratio, allowing conservation of the UDP-Glc donor and simplification of the purification process by minimizing excess reactants. ${ }^{94} \mathrm{Next}$, we desired to improve the product purification method. In our initial work from 2014, we had subjected the final reaction mixture to a precipitation step and then performed silica gel column chromatography using dichloromethane-methanol as the mobile phase to isolate the product. ${ }^{92}$ However, envisioning future scale-up needs, we wished to eliminate the silica gel chromatography and hazardous organic solvents. Based on the fact that, for reactions with quantitative conversion, the only neutral species remaining following the reaction incubation period is the desired product, we reasoned that we could apply ion exchange for improved purification. After significant testing and optimization, we found that we could process the crude reaction mixture through sequential (i) spin dialysis, to remove enzyme; and (ii) mixed-bed ion exchange resin treatment, to remove all ionic species in the mixture. ${ }^{93,94}$ This procedure left only the neutral trehalose analogue in aqueous solution, again assuming that the monosaccharide acceptor was quantitatively converted to product. During optimization, we also determined that switching the reaction buffer from HEPES to Tris- $\mathrm{HCl}$ led to cleaner purifications using the spin dialysis-ion exchange approach. For non-quantitative TreT reactions, after the spin dialysis and ion exchange steps, we applied P-2 gel column (100-1,800 MW fractionation range) using water as the mobile phase to separate unreacted monosaccharide from disaccharide product. ${ }^{93}$ Overall, following the one-step synthesis and all-aqueous purification process shown in 
Scheme 5B, we can now typically produce trehalose analogues in the 10-100 mg range in $\sim 60-80 \%$ isolated yield, and in a total duration of $\sim 30-120 \mathrm{~min} .{ }^{93}, 94$ The optimizations described here were reported in 2016 and 2017, ${ }^{93,} 94$ including a video protocol that facilitates rapid and straightforward synthesis of trehalose analogues by non-chemists. ${ }^{93}$

Despite the significant advantages the TreT method has for trehalose analogue synthesis, a number of limitations have spurred additional work to improve its efficiency and scope. One limitation is the expression yield of His-tagged TreT, which as noted above is $\sim 4 \mathrm{mg} / \mathrm{L}$ from E. coli lysate following $\mathrm{Ni}^{2+}$ affinity chromatography purification. To mitigate this issue, in 2018 we developed solid support-conjugated variants of TreT that we anticipated would enable catalyst recycling (Scheme 6). ${ }^{95}$ We conjugated purified His-tagged TreT to $N$ hydroxysuccinimide (NHS)-activated magnetic and agarose beads, which allowed postreaction enzyme capture using a magnetic tube rack or centrifugation, respectively. Both constructs retained enzymatic activity and provided effective TreT recycling, as quantitative conversion was observed over 10 successive rounds of synthesis. ${ }^{95}$ However, the agaroseimmobilized enzyme was less expensive and more robust, as we observed loss of magnetic beads after several rounds of recapture. ${ }^{95}$ Immobilized TreT will not only allow catalyst reuse, but may also be beneficial in automated/flow chemistry formats (e.g., see ${ }^{18} \mathrm{~F}$ radiosynthesis discussion below). In addition to TreT immobilization, we are also exploring solubility-tagged TreT constructs to increase protein yield, and our efforts so far have resulted in a novel construct that improves yields by 5 -fold (unpublished data).

Another area of interest is expanding the scope of the TreT reaction. While the native enzyme already possesses exceptional substrate tolerance in the acceptor variance mode, as noted above there are some substrates of interest that are not accepted by the enzyme, particularly 4-position analogues (Figure 6). Unfortunately, rational design of TreT mutants to improve substrate tolerance remains challenging due to the lack of a T. tenax TreT crystal structure. Thus, moving forward, it would be highly beneficial to generate a crystal structure of the enzyme to inform rational site-directed mutagenesis, as well as to pursue other strategies, such as directed evolution. In the meantime, we have also successfully expanded substrate scope by exploring TreT-catalyzed analogue synthesis in the donor variance mode, in which the UDP-sugar donor is altered instead of the acceptor (Scheme 7). This strategy, which we reported in 2018, provided efficient access to $2-\mathrm{N}$-modified trehalose analogues that were inaccessible via the acceptor variance mode. ${ }^{95}$

While we are currently pursuing various avenues to bolster the scale and scope of TreTbased chemoenzymatic synthesis, this and other chemoenzymatic methods have already accelerated the development of trehalose analogues for applications in microbiology and other fields. To illustrate this progress, in the following sections we tell three stories that highlight the role of chemoenzymatic synthesis in advancing trehalose analogues as bacterial imaging probes and metabolic inhibitors. Then, we close this article by recapping the progress made so far and discussing priorities for future research in this area, with an eye toward leveraging chemoenzymatic synthesis to continue expanding the functionality and applicability of trehalose analogues for the study and targeting of diverse bacterial pathogens. 


\section{Imaging mycobacteria with trehalose analogues}

In mycobacteria, trehalose is at the nexus of central carbon metabolism and cell envelope biosynthesis/recycling (see Figure 3). ${ }^{61}$ Trehalose is responsible for shuttling mycolic acids to the mycomembrane and is itself incorporated into glycolipids that reside in the mycomembrane via mycobacteria-specific pathways. ${ }^{37}$ Thus, there has been considerable interest in developing trehalose analogues modified with detectable groups that can hijack trehalose metabolic pathways and incorporate into the mycobacterial cell surface. ${ }^{73,96,97}$ Such detectable trehalose-based imaging probes have proven valuable for answering fundamental questions about mycobacterial cell envelope structure and metabolism, $60,65,66,98-107$ and are currently being pursued as tools for anti-mycobacterial drug development $^{47,108}$ and imaging of mycobacterial infections. ${ }^{94,109-111}$

Although progress on trehalose-based imaging probes over the last decade has been swift and exciting, a remaining impediment to the creation and adoption of these tools has been limited access to existing probes and the development of new probes. Chemoenzymatic synthesis has played an important role in addressing these problems, an illustrative example of which relates to the earliest trehalose analogues reported for imaging mycobacteria, FITC-Tre and the "clickable" TreAz series of analogues (structures shown above in Figure 4). FITC-Tre metabolically incorporates into trehalose glycolipids via substrate-promiscuous extracellular Ag85 enzymes and can be directly visualized, ${ }^{65}$ while TreAz analogues predominantly incorporate into trehalose glycolipids via the "inside-out" LpqY-SugABC recycling pathway and can be visualized on the cell surface following click reactions with (cyclo)alkyne-modified fluorophores ${ }^{66}$ (refer to Figure 3 for pathways). All of these probes were originally prepared by chemical synthesis. FITC-Tre was synthesized using the general approach shown in Scheme 1B, wherein custom building blocks were coupled via a specialized chemical glycosylation method, forging the a,a-1,1-glycosidic bond with high stereoselectivity. ${ }^{65}$ However, the longest linear sequence of this convergent synthesis was 8 steps and the overall yield was $<10 \%$, while the unnatural anomeric methyl group that controlled the glycosylation stereochemistry was present in the final product. ${ }^{65}$ All of the TreAz analogues were synthesized using the general approach shown in Scheme 1A, wherein trehalose was subjected to hydroxyl group protection/manipulation chemistries to arrive at the target compounds. ${ }^{66}$ The simplest target, 6-TreAz, took 4 steps to synthesize in $\sim 25 \%$ overall yield, while the syntheses for 2-, 3-, and 4-TreAz ranged from 6-9 steps and had overall yields of $<10 \% .{ }^{66}$ In addition to serving as imaging probes themselves, the TreAz analogues are convenient synthetic intermediates, as they can be elaborated to other structures by click chemistry with alkyne reagents or by azide reduction followed by treatment with amine-reactive reagents. For example, the latter approach was used to elaborate 2-, 3-, 4-, and 6-TreAz to the corresponding FITC-modified trehalose analogues 2-, 3-, 4- and 6-FlTre, which in total required 6-11 steps to synthesize from trehalose. ${ }^{99}$ All together, the high step counts, low overall yields, hazardous waste streams, and need for considerable synthetic expertise has hampered the chemical synthesis of fluorescent and clickable trehalose analogues.

While there have been no reports of OtsAB- or TreP-mediated syntheses of TreAz analogues or their derivatives, TreT catalysis can vastly improve the simplicity and efficiency of 
preparing and deploying this class of compounds (Scheme 8). We observed quantitative onestep conversion of commercially available 3- and 6-azido glucose (3- and 6-GlcAz) analogues to 3- and 6-TreAz, with isolated yields in the 60-80\% range using spin dialysision exchange purification (Scheme 8A). ${ }^{92,93}$ These structures can also be easily elaborated to 3- and 6-FITre by azide reduction and coupling with FITC, as shown previously (Scheme 8A). ${ }^{99}$ TreT-based routes are particularly beneficial for accessing 3-TreAz and 3-FITre, which by contrast require 8 and 10 steps to chemically synthesize from native trehalose, respectively. To exemplify how TreT can facilitate the application of trehalose-based imaging probes by non-chemists, we also demonstrated that 6-TreAz can be prepared using basic biological bench techniques and applied to metabolically label live mycobacteria in only $1 \mathrm{~h}$, a process which required only TreT enzyme and commercially available reagents. 92, 93 This approach has been adopted by other researchers to easily and rapidly access the probe for imaging experiments. ${ }^{112}$ As noted above, 4-position modifications are not welltolerated by TreT, and thus 4-TreAz and its derivatives were not accessible, reiterating this limitation of the T. tenax TreT enzyme. Interestingly, despite exhibiting otherwise excellent tolerance for 2-position modifications, TreT could not convert 2-GlcAz to 2-TreAz. ${ }^{92}$ Given the value of 2- $\mathrm{N}$-modified trehalose derivatives, including the imaging probes just described and 2-trehalosamine (2- $\mathrm{TreNH}_{2}$, an antibiotic discussed further below), coupled with the difficulty of their chemical synthesis (e.g., 2-TreAz requires 9 steps from trehalose ${ }^{66}$ ), we sought to identify an alternative TreT-based synthetic route for these structures. Our inability to identify a $2-\mathrm{N}$-modified acceptor substrate that was efficiently processed by TreT led us to explore whether the donor variance mode of synthesis might work instead (Schemes 7 and 8B). Gratifyingly, we found that TreT activity on UDP- $N$-acetyl-D-glucosamine (UDPGlcNAc) was equivalent to that of the native donor UDP-Glc, and we exploited this finding to synthesize the intermediate 2-TreNAc in $78 \%$ isolated yield. ${ }^{95}$ Subsequent $N$ deacetylation yielded 2- $\mathrm{TreNH}_{2}$, which was then elaborated to the imaging probes 2-FlTre and 2-TreAz via FITC coupling or diazo transfer, respectively. ${ }^{95}$ Thus, TreT catalysis delivered both $2-\mathrm{N}$-modified probes in 3 steps, whereas the same compounds required 9-11 steps via chemical synthesis. This study also revealed for the first time that TreT possesses substrate tolerance with respect to the UDP-sugar donor, which will be explored more fully in future work. T. tenax TreT catalysis was also used recently by the Fullam group to generate novel hydrolytically stable, epimeric TreAz analogues that can be used in conjunction with click chemistry to image mycobacterial glycolipids without the probes being channeled into degradative pathways (Figure 7). ${ }^{113}$ This study showed that TreT possesses tolerance for dual-modified acceptor substrates, and also established reaction conditions for producing 4-position-modified analogues with moderately improved yield. ${ }^{113}$ Overall, the TreT chemoenzymatic synthesis method has substantially improved the preparation of various fluorescent and clickable trehalose analogues for imaging mycobacteria.

Fluorescent- and click-tagged trehalose analogues have been extensively used for fluorescence imaging of live mycobacteria in cell culture. Some fluorogenic trehalose probes, synthesized via 6-TreAz as an intermediate, have even been shown to rapidly detect M. tuberculosis in patient sputum samples, demonstrating potential as point-of-care diagnostic tools. ${ }^{109,110}$ However, these probes rely on optical imaging and thus are not 
suitable for specific in vivo imaging of mycobacteria in patients. Such a capability is not yet available but could transform how M. tuberculosis infections are managed in the clinic, including challenging cases involving recurrence, drug resistance, or disseminated disease. 114-116 As originally suggested by Barry and Davis, ${ }^{65}$ trehalose analogues modified with a ${ }^{18} \mathrm{~F}$ radionuclide $\left({ }^{18} \mathrm{~F}\right.$-FDTre) could potentially enable in vivo visualization of mycobacteria using positron emission tomography (PET). PET is a clinically established imaging technique, usually used in conjunction with ${ }^{18} \mathrm{~F}$ radioprobes, which allows rapid, quantitative, three-dimensional imaging of metabolic processes deep within the human body. However, the synthesis of ${ }^{18} \mathrm{~F}$-FDTre analogues is a significant challenge due to the short half-life of ${ }^{18} \mathrm{~F}$ (108 min), which necessitates rapid synthesis and purification methods. ${ }^{117}$ Reported chemical syntheses of non-radioactive ${ }^{19} \mathrm{~F}-\mathrm{FDTre}$ analogues are lengthy and lowyielding, and the ${ }^{19} \mathrm{~F}$-fluorination steps in these reports involved long reaction times. $65,118,119$

Chemoenzymatic synthesis is particularly well-suited to the synthesis of ${ }^{18} \mathrm{~F}-\mathrm{FDTre}$ analogues. The standard PET radioprobe, 2-deoxy- $\left[{ }^{18} \mathrm{~F}\right]$ fluoro-D-glucose $\left({ }^{18} \mathrm{~F}-2-\mathrm{FDG}\right)$, is commercially available and used in a wide range of research and clinical PET imaging applications, including cancer imaging, neuroimaging, and cardiovascular imaging. $120{ }^{18} \mathrm{~F}-2$-FDG, and possibly other known ${ }^{18} \mathrm{~F}$-FDG positional isomers, ${ }^{121,} 122$ could be rapidly converted into ${ }^{18} \mathrm{~F}$-FDTre analogues via a chemoenzymatic approach. Indeed, as depicted above in Scheme 3, in 2011 Barry and Davis developed an OtsAB-inspired chemoenzymatic method for converting non-radioactive ${ }^{19} \mathrm{~F}-2$-FDG into ${ }^{19} \mathrm{~F}-2$-FDTre.${ }^{65}$ Although this initial ${ }^{19} \mathrm{~F}$ synthesis involved overnight reactions and HPLC purification, ${ }^{65}$ the method appears to be adaptable to ${ }^{18} \mathrm{~F}$ radiosynthesis. ${ }^{77}$ However, the OtsAB-based method is limited by the requirement for three separate enzymes, and to date has been used to synthesize only a single positional isomer.

Given the promising initial results showing that TreT can efficiently convert non-radioactive ${ }^{19} \mathrm{~F}$-FDG substrates to ${ }^{19} \mathrm{~F}$-FDTre analogue products in one step (Figure 6), ${ }^{92}$ we set out to further explore the potential of this system for developing ${ }^{18} \mathrm{~F}$-FDTre analogues as PET radioprobes. Our initial work in this area, published in 2016, focused on the TreT-mediated synthesis, conformational analysis, and mycobacterial uptake of non-radioactive ${ }^{19}$ F-FDTre analogues. ${ }^{94} \mathrm{~A}$ luminescence-based glycosyltransferase assay confirmed high activity of TreT on ${ }^{19} \mathrm{~F}-2-,{ }^{19} \mathrm{~F}-3$-, and ${ }^{19} \mathrm{~F}-6-\mathrm{FDG}$ ( $>85 \%$ activity relative to glucose), supporting the feasibility of our plan to exploit the wide availability of ${ }^{18} \mathrm{~F}-2$-FDG for future TreT-mediated synthesis of radioactive ${ }^{18} \mathrm{~F}$-2-FDTre. ${ }^{94}$ Using the optimized TreT one-step synthesis and all-aqueous purification process shown in Scheme $5 \mathrm{~B}$, we obtained pure ${ }^{19} \mathrm{~F}-2-,{ }^{19} \mathrm{~F}-3$-, and ${ }^{19}$ F-6-FDTre in 70-80\% isolated yield (Scheme 9). ${ }^{94}$ Consistent with other 4-positionmodified acceptor substrates, no TreT activity was observed for ${ }^{19} \mathrm{~F}-4-\mathrm{FDG}$, so we resorted to chemical synthesis to generate it in 5 steps from native trehalose. ${ }^{94}$ We performed structural characterization of the analogues by NMR and 2D NMR-informed molecular modeling, which showed that deoxyfluorination did not significantly impact the solution conformation of trehalose. ${ }^{94}$ Finally, we assayed analogue uptake in the model organism $M$. smegmatis, and found that ${ }^{19} \mathrm{~F}-2-,{ }^{19} \mathrm{~F}-3-$, and ${ }^{19} \mathrm{~F}-6$-FDTre, but not ${ }^{19} \mathrm{~F}-4-\mathrm{FDTre}$, actively accumulated in cells via the trehalose-specific transporter LpqY-SugABC, which is also present $M$. tuberculosis. ${ }^{94}$ This finding confirmed the presence of a conserved metabolic 
pathway in mycobacteria that specifically internalizes the probes. Together, this work established fundamental synthetic chemistry and microbial biochemistry on ${ }^{19} \mathrm{~F}-\mathrm{FDTre}$ analogues, directly supporting further development of ${ }^{18} \mathrm{~F}$-FDTre analogues as potential PET probes for in vivo imaging of mycobacterial infections.

We next worked to translate our findings on non-radioactive ${ }^{19} \mathrm{~F}$-FDTre analogues to radioactive ${ }^{18} \mathrm{~F}$-FDTre PET-capable radioprobes (Scheme 9). ${ }^{19} \mathrm{~F}$ chemistry is not trivial to adapt to ${ }^{18} \mathrm{~F}$ radiosynthesis, usually because achieving sufficiently short reaction and purification times is challenging. ${ }^{117}$ In the case of TreT catalysis, we had previously developed rapid processes, so the main concern was the acceptor substrate concentration. In our "cold" ${ }^{19}$ F-FDG reactions, we used $10-20 \mathrm{mM}$ of acceptor, however this is vastly higher than the trace picomolar concentrations of commercially available ${ }^{18} \mathrm{~F}-2$-FDG. Fortunately, this did not cause a problem and we avoided having to add non-radioactive substrate to increase concentration, which would have deleteriously reduced the specific activity of the radioprobe. The reactions were run essentially according to our established process for TreT catalysis, but using commercial ${ }^{18} \mathrm{~F}-2$-FDG as the acceptor substrate. Radio-HPLC analysis showed that complete conversion of ${ }^{18} \mathrm{~F}$-2-FDG to ${ }^{18} \mathrm{~F}$-2-FDTre was achieved in only 15 $\mathrm{min}$, and the spin dialysis-ion exchange purification was shortened to 15 min using cartridge-packed mixed-bed ion exchange resin. ${ }^{111}$ The target ${ }^{18} \mathrm{~F}$-2-FDTre radioprobe was obtained in high radiochemical yield and purity in only $30 \mathrm{~min}$, well within the timeframe needed for PET studies. Furthermore, in a collaboration with PET imaging company SOFIE, we performed a fully automated radiosynthesis of ${ }^{18} \mathrm{~F}$-2-FDTre using the commercial ELIXYS platform, ${ }^{111}$ which could be further streamlined with immobilized enzyme. ${ }^{95}$ Finally, to provide an initial gauge of ${ }^{18} \mathrm{~F}-2$-FDTre selectivity for bacteria, we measured its uptake in M. smegmatis and various mammalian cell lines. Promisingly, we found that $M$. smegmatis efficiently and specifically accumulated the radioprobe via trehalose metabolism, whereas no uptake was observed in any of the mammalian cell lines. ${ }^{111}$ This work, which was reported in 2019, established rapid and robust protocols for TreT-based radiosynthesis of ${ }^{18} \mathrm{~F}-2$-FDTre from a starting material that is universally available at radiopharmacies/PET facilities, and, furthermore, demonstrated the potential of ${ }^{18} \mathrm{~F}-2$-FDTre as a bacteria-specific PET radioprobe. ${ }^{111}$

The development of trehalose analogues for imaging mycobacteria is a rapidly evolving area of research that has been bolstered by new chemoenzymatic synthesis methods. We described here how the OtsAB and especially the TreT trehalose biosynthesis pathways have been exploited to improve the overall convenience and efficiency of synthesizing fluorescent and clickable trehalose analogues for in vitro imaging, and, moreover, how these methods allow unprecedented access to novel PET radioprobes for future in vivo imaging applications. For fluorescent and clickable probes, improving the scope and scale of existing chemoenzymatic synthesis methods and exploring other synthetic approaches (e.g., the inverting TreP system, Scheme 4) will be highly beneficial for the field. On the other hand, for the ${ }^{18} \mathrm{~F}$ PET radioprobes, scale is not an issue, and it is clear that TreT catalysis already offers a powerful way to produce ${ }^{18} \mathrm{~F}-2$-FDTre (and the 3 - and 6-position isomeric variants), so much of the remaining work will focus on establishing this tool for in vivo PET imaging of M. tuberculosis in animal models and potentially human patients. Although the impetus for FDTre development has so far centered on mycobacteria, numerous other types of 
bacteria possess machinery to internalize trehalose (Figures 2 and 3 and discussion thereof), so a key remaining objective is to establish the uptake selectivity profile of FDTre analogues in diverse bacteria. It is possible that isomeric ${ }^{18} \mathrm{~F}$-FDTre radioprobes will display divergent selectivity profiles that could be exploited to image different types of bacterial infections. Chemoenzymatic synthesis can be used to produce labeled trehalose reagents on demand to support these essential studies.

\section{Addressing drug-tolerant mycobacterial persisters with trehalose-based biofilm inhibitors}

Decades before trehalose analogues were conceived of as possible bacterial imaging probes, they had been shown to inhibit mycobacterial growth. Fascinatingly, 2-trehalosamine (2$\mathrm{TreNH}_{2}$ ) is a natural product that was originally isolated from Streptomyces in 1957 and shown to potently inhibit growth of $M$. tuberculosis (minimum inhibitory concentration (MIC) $\sim 6 \mu \mathrm{M}) .{ }^{123,}{ }^{124}$ However, no substantial follow-up studies were done on the antimycobacterial activity of 2-TreNH $\mathrm{N}_{2}$, likely because its isolation from Streptomyces is arduous ${ }^{123,124}$ and its previously reported chemical synthesis is lengthy and challenging. ${ }^{125}$ Synthetic trehalose analogues have also been explored as anti-mycobacterial compounds, with the first report in 1997 showing that 6-TreAz inhibited growth of M. aurum on solid medium with moderate potency (MIC $\sim 500 \mu \mathrm{M}$ ). ${ }^{49}$ Subsequent studies evaluating various trehalose analogues confirmed that the compounds display growth inhibition of mycobacteria with moderate potency (MICs generally $\geq 500 \mu \mathrm{M}$ ). ${ }^{65} \mathrm{TDM}$ analogues have also been prepared and tested for anti-mycobacterial activity. ${ }^{126-128}$

Several years ago, we became interested in applying trehalose analogues to investigate and target a newly identified connection between trehalose metabolism and mycobacterial biofilm formation. Mycobacterial biofilms grown in vitro model the so-called persister populations of mycobacteria that occur in vivo, which have extraordinary tolerance to antibiotics and are responsible for the lengthy tuberculosis treatment regimens (6 months or more), which ultimately contribute to acquired multidrug resistance. ${ }^{129}$ Thus, there is a great deal of interest in identifying mycobacterial biofilm inhibitors as possible persister-targeting adjunctive therapies that may reduce treatment durations and thus slow the emergence and spread of multidrug-resistant M. tuberculosis. ${ }^{130}$ Mycobacterial biofilms grown in vitro are rich in extracellular mycolic acids, suggesting a possible role for trehalose in biofilm formation due to its mycolic acid shuttling function. ${ }^{131}$ This link was first confirmed in a 2010 study by Ojha, which showed that the liberation of free extracellular mycolic acids from TDM by Tdmh was required for the formation of drug-tolerant biofilms in $M$. smegmatis (see Figure 3). ${ }^{53}$

Based on the work of Ojha on $\mathrm{Tdmh}^{53}, 54,132$ and our own preliminary observations that TreAz treatment of M. smegmatis induced a clumping phenotype indicative of cell wall modulation, we hypothesized that trehalose analogues would impair mycobacterial biofilm formation by inhibiting trehalose metabolism. To test this hypothesis, we designed a focused 8-member panel of trehalose analogues with modifications mainly at the 2- and 6-positions (Figure 8). ${ }^{133}$ These structures were prioritized because we observed in preliminary studies 
that 2- and 6-TreAz, but not 3- and 4-TreAz, inhibited M. smegmatis growth. ${ }^{66}$

Traditionally, chemical synthesis of the designed analogues would have been the bottleneck to hypothesis-testing, but we employed the TreT chemoenzymatic synthesis method to accelerate this step of the project. Using the optimized TreT process, we synthesized 7 out of the 8 compounds in good yield. ${ }^{133}$ While at the time, 2-TreAz required chemical synthesis according to a reported procedure ${ }^{66}$ since its corresponding azido glucose cannot serve as a TreT acceptor substrate (Figure 6), ${ }^{92}$ we have since developed a 3-step synthesis of 2-TreAz by exploiting the donor variance mode of TreT synthesis (Scheme 7). ${ }^{95}$ We assayed the 8 analogues in parallel for inhibition of $M$. smegmatis planktonic growth, in which bacteria are free-floating in liquid medium, and biofilm formation, in which bacteria are surfaceattached. The differences in activity were striking. While most of the compounds showed partial inhibition of planktonic growth at $500 \mu \mathrm{M}$, which was consistent with prior studies, several compounds completely blocked biofilm formation at 10-fold lower concentrations. $133 \mathrm{We}$ also found that all inhibitory activity was dependent on the trehalose-recycling transporter LpqY-SugABC (see Figure 3), confirming that the compounds exert their effects specifically on trehalose metabolism and that they must be taken up into the cell to do so. ${ }^{133}$ We speculated at the time ${ }^{133}$ that perhaps the anti-biofilm activity was due to inhibition of mycolic acid shuttling at some point following LpqY-SugABC-mediated uptake, which presumably would reduce the amount of free extracellular mycolic acids known to be required for biofilm formation in $M$. smegmatis. Regardless of the precise mechanism of action, these data, reported in 2017 , supported the hypothesis that trehalose analogues would selectively block biofilm formation in mycobacteria.

Given the promising selective anti-biofilm activity of trehalose analogues in the model organism M. smegmatis, we extended our studies to M. tuberculosis in collaborative work with the Eoh group, which was published in 2019. ${ }^{134}$ We chose to evaluate 6-TreAz in this study because (i) it selectively inhibited biofilm formation in M. smegmatis $^{133}$ and (ii) its 4step chemical synthesis from native trehalose could be scaled to meet the needs of the project. ${ }^{68}$ The latter point highlights the fact that relatively small scales (e.g., 10-100 mg for the TreT method) remain one of the key drawbacks of chemoenzymatic trehalose analogue synthesis, which must be addressed moving forward. It was found that 6-TreAz selectively inhibits $M$. tuberculosis biofilm formation, in a manner consistent with our findings in $M$. smegmatis. ${ }^{134}$ Surprisingly, in vitro enzyme inhibition and metabolomics experiments revealed that the likely mechanism of action of 6-TreAz was not direct inhibition of the mycolic acid shuttling cycle, as we initially anticipated, but rather competitive inhibition of intracellular TreS (see Figure 3). ${ }^{134}$ TreS interconverts trehalose and maltose, the latter of which can be elaborated to a-glucans or be channeled into central carbon metabolism. ${ }^{43}$ The model that emerged from this work suggested that as nutrient-deprived M. tuberculosis transitions from an actively replicating state to a biofilm state, cell wall trehalose glycolipids are broken down (e.g., by Tdmh) to release free trehalose, which is recycled via LpqYSugABC and then channeled via TreS into central carbon metabolism to support critically needed ATP and antioxidant synthesis. ${ }^{134}$ 6-TreAz interferes with this TreS-mediated trehalose catalytic shift, which appears to be an important adaptive response mechanism used by mycobacteria during stress to transition into a drug-tolerant persister state. To investigate the potential of trehalose analogues as adjunctive therapeutics, we performed 
combination treatment of M. tuberculosis with 6-TreAz and bedaquiline (BDQ), an antitubercular compound that targets ATP synthesis and was recently FDA-approved to treat multi-drug-resistant tuberculosis in the clinic. ${ }^{134}$ Excitingly, 6-TreAz treatment rapidly sensitized M. tuberculosis to BDQ both in broth culture and macrophage infection experiments, demonstrating the promise of trehalose analogues as lead compounds in this context. ${ }^{134}$

While there has been interest in the inhibitory properties of trehalose analogues for many years, our work developed the first trehalose-based biofilm inhibitors and established proofof-concept that these compounds may be valuable for targeting the drug-tolerant persister state of $M$. tuberculosis. The TreT chemoenzymatic synthesis method has been important to progress in this area, as it accelerated access to a small but diverse group of trehalose analogues for our initial biofilm screen in $M$. smegmatis. We expect that TreT catalysis will facilitate continued work to expand this compound class and apply it to M. tuberculosis. Chemoenzymatic synthesis may also help to revive the long-dormant research on trehalosebased aminoglycosides (e.g., 2-TreNH $\mathrm{N}_{2}$ ) as potentially more potent anti-mycobacterial compounds, an area we are actively pursuing. ${ }^{95}$ Continued improvement of the TreT method, and possibly engagement of complementary TreP- or OtsAB-based methods, will be important to drive progress in this area. In particular, as trehalose-based inhibitors advance to preclinical investigation, larger quantities of material than chemoenzymatic synthesis can currently deliver will be needed. This issue must be addressed through method improvements described above, including enhancing efficiency with respect to enzyme yield and substrate cost/availability.

\section{Inhibiting $C$. difficile trehalose utilization with degradation-resistant trehalose analogues}

Although the unique trehalose metabolic pathways of mycobacteria (Figure 3) have been the main focus of our work to date, numerous other microbes also have important trehaloserelated pathways (Figure 2 and related discussion) that can potentially be probed or blocked by trehalose analogues. To exemplify how trehalose analogues can more broadly impact bacteriology, we focus our last vignette on $C$. difficile, which causes a rapidly emergent and increasingly deadly intestinal infection. ${ }^{135}$ As described above, in 2018 it was reported that multiple epidemic $C$. difficile ribotypes (RT027, RT017, RT078) have evolved hyperefficient trehalose catabolic pathways that support bacterial growth on low levels of trehalose and may enhance the virulence of these strains. ${ }^{18,19}$ These pathways involve PTSmediated uptake of exogenous trehalose to form intracellular Tre-6-P, which is then hydrolyzed by the phosphotrehalase TreA to yield glucose and Glc-6-P (Figure 2B). ${ }^{18}, 19$

We hypothesized that trehalose analogues designed to resist trehalase-catalyzed hydrolysis would not be catabolized through the PTS-TreA pathway, and thus would (i) not promote growth of $C$. difficile and (ii) potentially inhibit utilization of exogenous native trehalose by C. difficile. ${ }^{136}$ When we initiated this project in 2018, two trehalase-resistant trehalose analogues had been previously characterized in the literature, the trehalose epimers LactoTre and MannoTre (structures shown in Figure 6). ${ }^{84,88,90}$ Interestingly, both compounds had 
been synthesized through enzymatic routes, with LactoTre being produced via inverting TreP catalysis in $40 \%$ yield $^{84}$ and MannoTre being produced via Thermotoga maritima TreT catalysis in $70 \%$ yield. ${ }^{90}$ In vitro enzyme experiments in these studies showed that LactoTre and MannoTre were resistant to hydrolysis by Bacillus trehalase (bacterial) and porcine kidney trehalase (mammalian), respectively. Neither compound was tested for inhibition of trehalose metabolism in bacterial cells. ${ }^{84, ~ 88,90}$ Since LactoTre and MannoTre were literature-established degradation-resistant analogues, we included them in our $C$. difficile study. We synthesized MannoTre in one step via T. tenax TreT catalysis in $72 \%$ yield, whereas LactoTre, which is inaccessible via TreT catalysis due to its 4-position modification, was chemically synthesized in 5 steps from trehalose. ${ }^{136}$ In addition, we sought to explore whether swapping the endocyclic oxygen for sulfur would yield a novel degradation-resistant trehalose analogue, as there was literature precedent that this modification in other types of glycosides conferred resistance to the cognate glycosidase enzyme. ${ }^{137}$ We designed 5-deoxy-5-thio-D-trehalose (5-ThioTre, structure shown in Figure 6), and chemoenzymatic synthesis was critical for the preparation of this compound. Whereas chemical synthetic routes to 5-ThioTre would likely be impractical, TreT catalysis delivered the compound in a single step in $77 \%$ yield from commercially available 5deoxy-5-thio-D-glucose. ${ }^{136}$

We evaluated the three synthesized trehalose analogues in enzymatic assays and $C$. difficile culture. All three compounds were confirmed to be resistant to hydrolysis by a model trehalase enzyme, porcine kidney trehalase. ${ }^{136}$ Furthermore, at least MannoTre and 5ThioTre inhibited hydrolysis of native trehalose by the trehalase, with the novel analogue 5ThioTre inhibiting trehalase activity with $\sim 10$-fold higher potency than the known analogue MannoTre. ${ }^{136}$ In collaboration with the Britton group, we next tested the degradationresistant analogues in $C$. difficile clinical isolates, including the epidemic trehalose-utilizing strains RT027 and RT078. In sole carbon source studies, $C$. difficile RT027 and RT078 could not grow on LactoTre or 5-ThioTre. Surprisingly, despite its resistance to, and inhibition of, a model trehalase enzyme, MannoTre supported growth of both strains at the same level as native trehalose, even when TreA was deleted. ${ }^{136}$ This result implied that MannoTre could be degraded by a different enzyme, perhaps an a-mannosidase. To determine whether the analogues blocked RT027 and RT078 from utilizing trehalose from their environment, the strains were incubated in the presence of native trehalose as the sole carbon source along with varying concentrations of the analogues, and growth was assessed. MannoTre enhanced growth moderately, LactoTre inhibited growth of RT027 but not RT078, and 5-ThioTre inhibited growth of both ribotypes. ${ }^{136}$ Thus, the compounds exhibited intriguingly divergent activities, with the novel analogue 5-ThioTre demonstrating the broadest effectiveness as an inhibitor of $C$. difficile trehalose utilization.

Together, the initial discovery of $C$. difficile trehalose metabolism variants in 2018 and follow-up studies, including our above-described work on trehalose analogues reported in 2019, have a number of important implications. It was originally suggested in 2018 that the widespread introduction of trehalose into the human diet as a food additive may have promoted the evolution, spread, and virulence of $C$. difficile ribotypes that metabolize trehalose hyper-efficiently. ${ }^{18,19}$ The possibility that dietary trehalose dysregulates the gut microbiota and compromises human health led us to explore degradation-resistant trehalose 
analogues as tools to investigate and mitigate this potential problem. We capitalized on TreT chemoenzymatic synthesis to identify 5-ThioTre as a novel and promising analogue. 5ThioTre is not only trehalase-resistant, but it is also the most potent trehalose-based trehalase inhibitor reported to date and was the only compound that prevented trehalose utilization in all $C$. difficile strains tested. As discussed above, additional investigation is needed to determine the extent to which trehalose metabolism is involved in $C$. difficile virulence and whether it is indeed a good target for drug development. Regardless, degradation-resistant trehalose analogues are valuable in several other contexts. Numerous types of bacteria possess trehalose catabolic pathways comprising transporter-trehalase systems that, analogous to the $C$. difficile PTS-TreA system, support bacterial growth and can conceivably be targeted by trehalose analogues. Consistent with this notion, our data described above suggest that 6-TreAz inhibits mycobacterial biofilm formation by targeting the comparable LpqY-SugABC-TreS system. ${ }^{134}$ Thus, there is strong justification to evaluate degradation-resistant analogues as trehalose metabolism inhibitors in other bacterial pathogens, as well as in other trehalose-utilizing pathogens or pests, such as fungi and insects. ${ }^{13,138,139}$

Degradation-resistant trehalose analogues have other applications as well. As noted in the introductory overview of trehalose, the native disaccharide is being pursued as a therapeutic for various ailments, including cardiometabolic, neurodegenerative, and viral diseases. However, the efficacy of trehalose in these contexts is limited because it can be hydrolyzed by human-resident endogenous and/or microbial trehalases, thus drastically reducing its bioavailability. In principle, trehalose analogues that mimic trehalose's structure and beneficial bioactivity, but resist enzymatic hydrolysis, can solve the bioavailability problem while simultaneously alleviating concerns about the growth or virulence of host microbial populations. In pursuit of this goal, we have worked in collaboration with the DeBosch group to develop degradation-resistant LactoTre as a potential therapeutic for treating cardiometabolic diseases. ${ }^{24,140-142}$ Excitingly, in comparison to native trehalose, LactoTre exerts comparable or enhanced metabolic effects without contributing to $C$. difficile RT027 growth or virulence in vivo. ${ }^{24,142}$ To support these efforts from the synthetic side, we initially produced LactoTre via a 5 -step chemical synthesis route because this analogue is inaccessible via TreT catalysis. However, as experimentation progressed to animal models, demand for LactoTre increased and scale-up was essential. To meet this demand, Hayashibara Co. was enlisted to synthesize LactoTre on gram scale using the TreP catalysis method of Chaen et al., which features in situ generation of the $\beta$-Glc-1-P donor substrate. ${ }^{84}$ As we work to expand degradation-resistant trehalose analogues and apply them in various systems of interest, chemoenzymatic synthesis will continue to be pivotal, both for initial compound evaluation and subsequent scale-up. As with our work on biofilm inhibitors, we anticipate that the TreT chemoenzymatic synthesis method, in its current form, will provide rapid access to diverse and novel analogues for initial testing (e.g., 5-ThioTre), while scaleoptimized TreT or TreP methods will be required to advance prioritized compounds to preclinical or clinical studies. 


\section{Conclusions and outlook}

Trehalose, historically known for its bioprotective properties, has emerged as a promising target for antibiotic and diagnostic development due to its importance to bacterial virulence and its absence from humans. Two examples of trehalose-utilizing pathogens that have a major global health impact are M. tuberculosis and $C$. difficile, although numerous other bacterial pathogens possess trehalose metabolic pathways as well. As depicted in Figures 2 and 3, the metabolic pathways bacteria employ to synthesize, degrade, and otherwise use trehalose can vary significantly between species, but the extent to which these pathways contribute to virulence is still not clear in many cases. New tools are needed to facilitate the elucidation of bacterial trehalose metabolism pathways, probe their roles in virulence, and leverage them to improve the detection and treatment of bacterial infections. Over the past decade, synthetic trehalose analogues have emerged as linchpin tools in the pursuit of understanding and targeting bacterial trehalose metabolism. In the early 2010s, fluorescent and clickable trehalose analogues were introduced and the value of these compounds for imaging live mycobacteria was immediately apparent. We recognized that the challenges associated with the synthesis of these probes would impede the adoption of these tools by the microbiology community and would slow the development and applications of novel trehalose-based probes and inhibitors. Trehalose's 1,1-a,a-glycosidic bond and $C_{2}$ symmetry make analogue synthesis via traditional chemical approaches challenging, so we turned to chemoenzymatic synthesis methods that are operationally simple, fast, and highyielding. While some progress had been made in this area by exploiting the OtsAB and TreP pathways for analogue synthesis, these methods have various limitations for synthetic applications, relatively few analogues have been made, and little work has been aimed at making tools for bacteriology. In 2014, we introduced a chemoenzymatic synthetic method that exploits the substrate-tolerant, thermostable TreT enzyme from T. tenax. This method was iteratively refined in subsequent years, ultimately leading to a convenient and robust process for the rapid one-step synthesis and all-aqueous purification of diverse trehalose analogues.

We have employed the TreT method as a platform to both improve the synthesis of known trehalose analogues and develop new analogues with novel function, generally with the goal of creating and increasing the accessibility of tools that facilitate the study and targeting of bacterial trehalose metabolism. Fluorescent and clickable trehalose analogues that have proven useful for the mycobacteria field can now be generated in 1-3 steps and high overall yield via TreT catalysis, rather than 4-11 steps and low overall yield via chemical synthesis. In addition to improving syntheses of existing trehalose-based probes, TreT catalysis has enabled the development of next-generation probes. For example, we have applied TreT to facilitate the development of ${ }^{18} \mathrm{~F}$-FDTre analogues as potential PET probes for in vivo imaging of mycobacterial infection. TreT catalysis has proven particularly useful for this application, as it first delivered several non-radioactive ${ }^{19} \mathrm{~F}$-FDTre analogues to perform feasibility studies, and was then successfully adapted to the rapid, automated one-step radiosynthesis of ${ }^{18} \mathrm{~F}$-2-FDTre starting from the commercially available PET probe ${ }^{18} \mathrm{~F}-2$ FDG. In the realm of inhibitor development, TreT catalysis was instrumental in quickly accessing a small panel of compounds that were used to test and confirm the hypothesis that 
trehalose analogues would inhibit mycobacterial biofilm formation and sensitize $M$. tuberculosis biofilms to existing anti-tubercular compounds. Similarly, we deployed TreT catalysis to produce three degradation-resistant trehalose analogues, including the novel resistant analogue 5-ThioTre, and showed that this sulfur-modified analogue blocked trehalose metabolism in multiple epidemic strains of $C$. difficile, a major intestinal pathogen. Overall, the optimized TreT chemoenzymatic synthesis process has been successful in accelerating the development, evaluation, and adoption of trehalose-based imaging probes and inhibitors.

With respect to future work, there are numerous research directions that need to be pursued in the areas of improving synthetic methods, answering fundamental questions about bacterial trehalose metabolism, and advancing the development of trehalose analogues as clinically relevant imaging probes and inhibitors. Chemoenzymatic synthesis methods, particularly TreT catalysis, have proven useful for synthesizing valuable functionalized trehalose analogues, but the accessible structures are relatively simple (e.g., mono- or disubstituted analogues) and are typically obtained in moderate scales (typically 10-100 $\mathrm{mg}$ ). The scope of these synthetic approaches needs to be expanded, both in terms of further testing/improving substrate tolerance of trehalose-synthesizing enzymes and also adding trehalose-modifying enzymes to the toolbox. There has been some success in engineering inverting TreP enzymes to expand substrate scope, and we are pursuing similar strategies to accomplish this for TreT. This work will be bolstered if a T. tenax TreT crystal structure can be obtained. Mining microbial genomes for other trehalose-synthesizing enzymes may also identify new catalysts with improved or complementary substrate tolerance. In addition, many naturally occurring trehalose derivatives have hydroxyl groups modified by acylation, sulfation, phosphorylation, or glycosylation. Identifying enzymes that can robustly and siteselectively install these groups for synthetic purposes would be highly valuable. For example, we have developed a class of TMM- and TDM-based probes for mycobacteria, 98, 104-107 whose syntheses could potentially be improved by enlisting existing methods for direct enzymatic 6- $O$-acylation of native trehalose. ${ }^{143,144}$ Reaction scale also remains a significant impediment, as illustrated by our need to prioritize M. tuberculosis biofilm inhibitor evaluation based on synthetic scale and not necessarily compound activity. For the TreT method, this means enhancing efficiency of enzyme production and addressing cases where substrate cost or availability are an issue. Promisingly, industrial-scale production of native trehalose via a TreYZ-based method and gram-scale production of the trehalose analogue LactoTre via a modified TreP method have been successful, suggesting that scale up of other chemoenzymatic methods, including TreT catalysis, is feasible.

As synthetic methods mature and trehalose-based probes and inhibitors expand in functionality and accessibility, these tools can continue to be harnessed to improve our understanding of bacterial trehalose metabolism. Trehalose analogues have already driven forward our understanding of mycobacterial cell envelope biosynthesis and remodeling, although much remains to be learned in this regard, particularly with respect to the metabolism, spatiotemporal dynamics, and host interactions of virulence-associated trehalose glycolipids. Looking beyond mycobacteria, there is a frontier in terms of elucidating bacterial trehalose metabolic pathways and defining their roles in virulence, as underscored by the groundbreaking reports on $C$. difficile trehalose utilization that occurred 
over just the past two years. There is evidence, mainly from sole carbon source studies, that many other types of bacteria have the ability to metabolize trehalose. This is further supported by genomic data. For instance, trehalose transporters (annotated) are common in human gut microbiome metagenomes. However, in the vast majority of cases, the molecular players, efficiencies, and physiological roles of these trehalose uptake pathways are not known. Determining these aspects of bacterial trehalose metabolism may lead to new strategies for detecting and treating bacterial infections. Indeed, we have already demonstrated proof-of-concept by successfully targeting the mycobacterial trehalose transporter, LpqY-SugABC, with imaging probes and metabolic inhibitors. We anticipate that chemoenzymatically synthesized trehalose analogues, particularly detectable probes that can be deployed in live bacteria, will be critical to supporting these lines of fundamental research.

Finally, we highlighted several areas where chemoenzymatically synthesized trehalose analogues have considerable potential to be advanced as candidates for imaging or treating bacterial infections. There is an unmet clinical need to image bacterial infections within human patients. Targeting bacterial-specific carbohydrate metabolism, for example by using ${ }^{18}$ F-FDTre PET radioprobes, is a promising approach. Although we have established a robust chemoenzymatic method for synthesizing ${ }^{18} \mathrm{~F}-\mathrm{FDTre}$ analogues and demonstrated efficient uptake by mycobacteria, much work remains to be done to establish the utility of ${ }^{18}$ F-FDTre for preclinical or clinical infection imaging. For example, defining the uptake kinetics of the probes in different types of bacterial and host cells is a critical next step because it will inform the probes' applications and limitations, and it will facilitate the design and interpretation of future imaging studies in animal models and possibly humans. Given the importance of trehalose metabolism to the growth and/or virulence of various bacterial pathogens, small molecules that inhibit these pathways may have therapeutic potential. Toward this goal, we have enlisted TreT catalysis to drive progress in the development of different classes of trehalose-based metabolic inhibitors. In most cases, these efforts are in their early stages, although we have demonstrated promising inhibitory activity in major pathogens, including $M$. tuberculosis and $C$. difficile. Notably, we identified a trehalose-based mycobacterial biofilm inhibitor that sensitizes drug-tolerant $M$. tuberculosis to the clinical anti-tubercular compound BDQ, which has implications for the development of urgently needed short-course combination therapies for tuberculosis treatment. Moving forward, we need to expand the number of analogues being assayed to identify more potent inhibitors, as well as develop scalable syntheses to support mechanism-of-action and preclinical studies. Ultimately, as we and others seek to develop and apply trehalose analogues to achieve the synergistic goals of (i) better characterizing bacterial trehalose metabolic pathways and (ii) exploiting these pathways to visualize or treat bacterial infections, chemoenzymatic synthesis will continue to play an essential role.

\section{Acknowledgements}

This work was funded by a grant to B.M.S. and P.J.W. from the National Institutes of Health (R15 AI117670), as well as a Henry Dreyfus Teacher-Scholar Award to B.M.S from The Camille \& Henry Dreyfus Foundation (TH-17-034). We thank our colleagues who collaborated with us on projects discussed in the article, including Dr. Brian DeBosch (Washington University School of Medicine), Dr. Robert Britton (Baylor College of Medicine), Dr. Hyungjin Eoh (University of Southern California Keck School of Medicine), Dr. Chris Drake (SOFIE Co.), and Dr. 
Juan Vaquero (Universidad Carlos III de Madrid). Dr. Brian DeBosch is thanked for reading and commenting on the manuscript. Noah Danielson is thanked for contributing to the table of contents graphic art.

\section{About the authors}

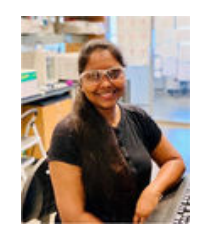

Karishma Kalera

Karishma grew up in Rajasthan, India, where she received a B.Sc. in Biology from Government College Ajmer in 2011 and a M.Sc. in Microbiology from Maharshi Dayanand Saraswati University in 2013. She worked as a lecturer in the USB College of Physiotherapy/Nursing and in a tuberculosis culture laboratory in India. In 2017, she was awarded a National Overseas Scholarship from the Government of India to support Ph.D. studies abroad. In 2018, she started her doctoral studies at Central Michigan University in the lab of Dr. Ben Swarts. Karishma's research is focused on the development of trehalosamine inhibitors of mycobacterial growth and biofilm formation.

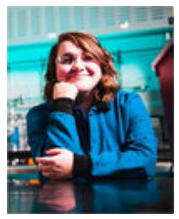

Alicyn I. Stothard

Alicyn is from Coleman, Michigan. She completed a B.S. in Biochemistry from Central Michigan University in 2020. She has worked in the lab of Dr. Ben Swarts since 2016. Her research mainly focused on the development of novel trehalose analogues that resist hydrolysis by trehalase enzymes. She has collaborated with investigators at CMU and other institutions to explore the applications of these compounds as therapeutics for human metabolic diseases and as inhibitors of trehalose metabolism in bacteria and insects. Among various recognitions for her undergraduate research accomplishments, Alicyn received the CMU President's Award for Outstanding Undergraduate Research \& Creative Accomplishments.

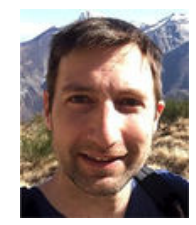

Peter J. Woodruff 
Pete was born in Philadelphia, PA. He graduated from Dartmouth College with a B.A. in Biophysical Chemistry in 1999. He earned a Ph.D. in Molecular and Cell Biology from the University of California, Berkeley, with Prof. Carolyn Bertozzi, studying the role of trehalose in the cell wall of mycobacteria. Since joining the faculty at the University of Southern Maine, Pete's research program initially focused on engineering bacteria for increased stress tolerance in order to expedite bioremediation of toxic metals. He received the Cottrell College Science Award, and has returned to studying the role of trehalose in pathogenic bacteria.

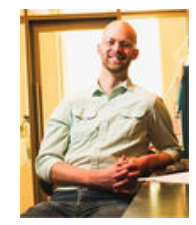

Benjamin M. Swarts

Ben grew up in Danville, Ohio. He completed a B.A. in Chemistry from the College of Wooster in 2004 and a Ph.D. in Chemistry from Wayne State University in 2010 with Prof. Zhongwu Guo, studying glycosylphosphatidylinositol anchors. After a postdoc with Prof. Carolyn Bertozzi at the University of California, Berkeley, in 2013 he joined the faculty at Central Michigan University. His research focuses on the synthesis of bacterial carbohydrates and the development of probes to investigate the mycobacterial outer membrane. Among other recognitions, Ben received the Cottrell College Science Award, the Henry Dreyfus Teacher-Scholar Award, and the NSF CAREER award.

\section{Notes and references}

1. Elbein AD, Pan YT, Pastuszak I and Carroll D, Glycobiology, 2003, 13, 17R-27R.

2. Ohtake S and Wang YJ, J. Pharm. Sci, 2011, 100, 2020-2053. [PubMed: 21337544]

3. Olsson C, Genheden S, García Sakai V and Swenson J, J. Phys. Chem. B, 2019, 123, 3679-3687. [PubMed: 30964287]

4. Hosseinpour-Moghaddam K, Caraglia M and Sahebkar A, J. Cell Physiol, 2018, 233, 6524-6543. [PubMed: 29663416]

5. Zhang Y and DeBosch BJ, Curr. Opin. Clin. Nutr. Metab. Care, 2019, 22, 303-310. [PubMed: 31033580]

6. Maruta K, Nakada T, Kubota M, Chaen H, Sugimoto T, Kurimoto M and Tsujisaka Y, Biosci. Biotechnol. Biochem, 1995, 59, 1829-1834. [PubMed: 8534970]

7. Giaever HM, Styrvold OB, Kaasen I and Strøm AR, J. Bacteriol, 1988, 170, 2841-2849. [PubMed: 3131312]

8. Horlacher R, Uhland K, Klein W, Ehrmann M and Boos W, J. Bacteriol, 1996, 178, 6250. [PubMed: 8892826]

9. Boos W, Ehmann U, Bremer E, Middendorf A and Postma P, J. Biol. Chem, 1987, 262, 1321213218. [PubMed: 2820965]

10. Rimmele M and Boos W, J. Bacteriol, 1994, 176, 5654-5664. [PubMed: 8083158]

11. Boos W, Ehmann U, Forkl H, Klein W, Rimmele M and Postma P, J. Bacteriol, 1990, 172, 34503461. [PubMed: 2160944]

12. Styrvold OB and Strøm AR, J. Bacteriol, 1991, 173, 1187. [PubMed: 1825082]

13. Thammahong A, Puttikamonkul S, Perfect JR, Brennan RG and Cramer RA, Microbiol. Mol. Biol. Rev, 2017, 81, e00053-00016. [PubMed: 28298477] 
14. Chouikha I, Germon P, Brée A, Gilot P, Moulin-Schouleur M and Schouler C, J. Bacteriol, 2006, 188, 977. [PubMed: 16428402]

15. Pavanelo DB, Houle S, Matter LB, Dozois CM and Horn F, Infect. Immun, 2018, 86, e0024100218. [PubMed: 29844238]

16. Vanaporn M, Sarkar-Tyson M, Kovacs-Simon A, Ireland PM, Pumirat P, Korbsrisate S, Titball RW and Butt A, Virulence, 2017, 8, 30-40. [PubMed: 27367830]

17. Schwarz S and Dijck PV, Virulence, 2016, DOI: 10.1080/21505594.2016.1216295.

18. Collins J, Danhof H and Britton RA, Gut Microbes, 2018, DOI: 10.1080/19490976.2018.1491266.

19. Collins J, Robinson C, Danhof H, Knetsch CW, van Leeuwen HC, Lawley TD, Auchtung JM and Britton RA, Nature, 2018, 553, 291-294. [PubMed: 29310122]

20. Helfert C, Gotsche S and Dahl MK, Mol. Microbiol, 1995, 16, 111-120. [PubMed: 7651129]

21. Schöck F and Dahl MK, J. Bacteriol, 1996, 178, 4576-4581. [PubMed: 8755887]

22. Dahl MK, Microbiol. Immunol, 1997, 148, 233-238.

23. Abt MC, Cell Host Microbe, 2018, 23, 156-158. [PubMed: 29447694]

24. Zhang Y, Shaikh N, Ferey JL, Wankhade UD, Chintapalli SV, Higgins CB, Crowley JR, Heitmeier MR, Stothard AI, Mihi B, Good M, Higashiyama T, Swarts BM, Hruz PW, Shankar K, Tarr PI and DeBosch BJ, Gastroenterology, 2020, 158, 1402-1416.e1402. [PubMed: 31838076]

25. Eyre DW, Didelot X, Buckley AM, Freeman J, Moura IB, Crook DW, Peto TEA, Walker AS, Wilcox MH and Dingle KE, EBioMedicine, 2019, 43, 347-355. [PubMed: 31036529]

26. Saund K, Rao K, Young VB and Snitkin ES, Open Forum Infectious Diseases, 2020, 7. DOI: 10.1093/ofid/ofz548.

27. Ells TC and Hansen LT, Appl. Environ. Microbiol, 2011, 77, 6841-6851. [PubMed: 21821737]

28. Vitko NP, Grosser MR, Khatri D, Lance TR and Richardson AR, mBio, 2016, 7, e00296-16. [PubMed: 27329749]

29. Murdoch DA and Mitchelmore IJ, J. Med. Microbio, 1991, 34, 295-308.

30. Chen YS, Srionnual S, Onda T and Yanagida F, Lett. Appl. Microbiol, 2007, 45, 190-193. [PubMed: 17651217]

31. Park M, Mitchell WJ and Rafii F, Int. J. Microbiol, 2016, DOI: 10.1155/2016/4829716.

32. Postma PW, Keizer HG and Koolwijk P, J. Bacteriol, 1986, 168, 1107-1111. [PubMed: 3023298]

33. Bidossi A, Mulas L, Decorosi F, Colomba L, Ricci S, Pozzi G, Deutscher J, Viti C and Oggioni MR, PLoS ONE, 2012, 7, e33320. [PubMed: 22428019]

34. Duong T, Barrangou R, Russell WM and Klaenhammer TR, Appl. Environ. Microbiol, 2006, 72, 1218-1225. [PubMed: 16461669]

35. Saubolle M. a. and Sussland D, J. Clin. Microbiol, 2003, 41, 4497-4501. [PubMed: 14532173]

36. World Health Organization, Global Tuberculosis Report 2019.

37. Quémard A, Trends in Microbiology, 2016, 24, 725-738. [PubMed: 27268593]

38. Marrakchi H, Lanéelle M-A and Daffé M, Chem. Biol, 2013, 21, 67-85. [PubMed: 24374164]

39. De Smet KAL, Weston A, Brown IN, Young DB and Robertson BD, Microbiology, 2000, 146, 199-208. [PubMed: 10658666]

40. Woodruff PJ, Carlson BL, Siridechadilok B, Pratt MR, Senaratne RH, Mougous JD, Riley LW, Williams SJ and Bertozzi CR, J. Biol. Chem, 2004, 279, 28835-28843. [PubMed: 15102847]

41. Murphy HN, Stewart GR, Mischenko VV, Apt AS, Harris R, McAlister MSB, Driscoll PC, Young DB and Robertson BD, J. Biol. Chem, 2005, 280, 14524-14529. [PubMed: 15703182]

42. Carroll JD, Pastuszak I, Edavana VK, Pan YT and Elbein AD, FEBS J, 2007, 274, 1701-1714. [PubMed: 17319935]

43. Miah F, Koliwer-Brandl H, Rejzek M, Field Robert A., Kalscheuer R and Bornemann S, Chem. Biol, 2013, 20, 487-493. [PubMed: 23601637]

44. Sambou T, Dinadayala P, Stadthagen G, Barilone N, Bordat Y, Constant P, Levillain F, Neyrolles O, Gicquel B, Lemassu A, Daffé M and Jackson M, Mol. Microbiol, 2008, 70, 762-774. [PubMed: 18808383]

45. Gavalda S, Bardou F, Laval F, Bon C, Malaga W, Chalut C, Guilhot C, Mourey L, Daffé M and Quémard A, Chem. Biol, 2014, 21, 1660-1669. [PubMed: 25467124] 
46. Grzegorzewicz AE, Pham H, Gundi VAKB, Scherman MS, North EJ, Hess T, Jones V, Gruppo V, Born SEM, Kordulakova J, Chavadi SS, Morisseau C, Lenaerts AJ, Lee RE, McNeil MR, Jackson M, Korduláková J, Chavadi SS, Morisseau C, Lenaerts AJ, Lee RE, McNeil MR and Jackson M, Nat. Chem. Biol, 2012, 8, 334-341. [PubMed: 22344175]

47. Xu Z, Meshcheryakov VA, Poce G and Chng S-S, Proc. Natl. Acad. Sci. U. S. A, 2017, 114, 79937998. [PubMed: 28698380]

48. Sathyamoorthy N and Takayama K, J. Biol. Chem, 1987, 262, 13417-13423. [PubMed: 3654621]

49. Belisle JT, Vissa VD, Sievert T, Takayama K, Brennan PJ and Besra GS, Science, 1997, 276, 1420-1422. [PubMed: 9162010]

50. Dautin N, de Sousa-d'Auria C, Constantinesco-Becker F, Labarre C, Oberto J, de la Sierra-Gallay IL, Dietrich C, Issa H, Houssin C and Bayan N, Biochim. Biophys. Acta, 2016, 1861, 3581-3592.

51. Hunter RL, Venkataprasad N and Olsen MR, Tuberculosis, 2006, 86, 349-356. [PubMed: 16343989]

52. Welsh KJ, Hunter RL and Actor JK, Tuberculosis, 2013, 93, Suppl, S3-S9. [PubMed: 24388646]

53. Ojha AK, Trivelli X, Guerardel Y, Kremer L and Hatfull GF, J. Biol. Chem, 2010, 285, 1738017389. [PubMed: 20375425]

54. Yang Y, Kulka K, Montelaro RC, Reinhart TA, Sissons J, Aderem A and Ojha AK, Cell Host Microbe, 2014, 15, 153-163. [PubMed: 24528862]

55. Middlebrook G, Coleman CM and Schaefer WB, Proc. Nat. Acad. Sci. U. S. A, 1959, 45, 18011804.

56. Ortalo-Magne A, Lemassu A, Laneelle MA, Bardou F, Silve G, Gounon P, Marchal G and Daffe M, J. Bacteriol, 1996, 178, 456-461. [PubMed: 8550466]

57. Burbaud S, Laval F, Lemassu A, Daffé M, Guilhot C and Chalut C, Cell Chem. Biol, 2016, 23, 278-289. [PubMed: 27028886]

58. Burguière A, Hitchen PG, Dover LG, Kremer L, Ridell M, Alexander DC, Liu J, Morris HR, Minnikin DE, Dell A and Besra GS, J. Biol. Chem, 2005, 280, 42124-42133. [PubMed: 16257960]

59. Kalscheuer R, Weinrick B, Veeraraghavan U, Besra GS and Jacobs WR Jr., Proc. Natl. Acad. Sci. USA, 2010, 107, 21761-21766. [PubMed: 21118978]

60. Pohane AA, Carr CR, Garhyan J, Swarts BM and Siegrist MS, bioRxiv, 2019, DOI: 10.1101/758177.

61. Kalscheuer R and Koliwer-Brandl H, Microbiology Spectrum, 2014, 2. DOI: 10.1128/ microbiolspec.MGM2-0002-2013

62. North EJ, Jackson M and Lee RE, Curr. Pharm. Des, 2014, 20, 4357-4378. [PubMed: 24245756]

63. Thanna S and Sucheck SJ, Med. Chem. Commun, 2015, 7, 69-85.

64. Reinink P, Buter J, Mishra VK, Ishikawa E, Cheng T.-y., Willemsen PTJ and Porwollik S, J. Exp. Med, 2019, 216, 757-771. [PubMed: 30804000]

65. Backus KM, Boshoff HI, Barry CS, Boutureira O, Patel MK, D’Hooge F, Lee SS, Via LE, Tahlan K, Barry CE 3rd and Davis BG, Nat. Chem. Biol, 2011, 7, 228-235. [PubMed: 21378984]

66. Swarts BM, Holsclaw CM, Jewett JC, Alber M, Fox DM, Siegrist MS, Leary JA, Kalscheuer R and Bertozzi CR, J. Am. Chem. Soc, 2012, 134, 16123-16126. [PubMed: 22978752]

67. Sletten EM and Bertozzi CR, Angew. Chem. Int. Edit, 2009, 48, 6974-6998.

68. Hanessian S and Lavallee P, J. Antibiot, 1972, 25, 683-684.

69. Sarpe VA and Kulkarni SS, Trends Carbohydr. Res, 2013, 5, 8-33.

70. Chaube MA and Kulkarni SS, Trends Carbohydr. Res, 2013, 4, 1-19.

71. Jana S and Kulkarni SS, Org. Biomol. Chem, 2020, 18, 2013-2037. [PubMed: 32115587]

72. Walmagh M, Zhao R and Desmet T, Int. J. Mol. Sci, 2015, 16, 13729-13745. [PubMed: 26084050]

73. O’Neill MK, Piligian BF, Olson CD, Woodruff PJ and Swarts BM, Pure Appl. Chem, 2017, 89, 1223-1249. [PubMed: 29225379]

74. Teramoto N, Sachinvala ND and Shibata M, Molecules, 2008, 13, 1773-1816. [PubMed: 18794785] 
75. Yamamoto T, Maruta K, Watanabe H, Yamashita H, Kubota M, Fukuda S and Kurimoto M, Biosci. Biotechnol. Biochem, 2001, 65, 1419-1423. [PubMed: 11471747]

76. Chenault HK, Mandes RF and Hornberger KR, J. Org. Chem, 1997, 62, 331-336. [PubMed: 11671407]

77. Conference Proceedings abstract in: J. Label. Compd. Radiopharm 2015, 58, S75-S411..

78. Luley-Goedl C and Nidetzky B, Biotechnol. J, 2010, 5, 1324-1338. [PubMed: 21154671]

79. Belocopitow E and Maréchal LR, Biochim. Biophys. Acta, 1970, 198, 151-154. [PubMed: 5413942]

80. Belocopitow E, Marechal LR and Gros EO, Carbohyd. Res, 1971, 19, 268-271.

81. Maruta K, Watanabe H, Nishimoto T, Kubota M, Chaen H, Fukuda S, Kurimoto M and Tsujisaka Y, J. Biosci. Bioeng, 2006, 101, 385-390. [PubMed: 16781466]

82. Van der Borght J, Chen C, Hoflack L, Van Renterghem L, Desmet T and Soetaert W, Appl. Environ. Microb, 2011, 77, 6939-6944.

83. Van der Borght J, Soetaert W and Desmet T, Biotechnol. Progr, 2012, 28, 1257-1262.

84. Chaen H, Nakada T, Mukai N, Nishimoto T, Fukuda S, Sugimoto T, Kurimoto M and Tsujisaka Y, J. Appl. Glycosci, 2001, 48, 135-137.

85. Chen C, Van der Borght J, De Vreese R, D'Hooghe M, Soetaert W and Desmet T, Chem. Commun, 2014, 50, 7834-7836.

86. Van der Borght J, Desmet T and Soetaert W, Biotechnol. J, 2010, 5, 986-993. [PubMed: 20799297]

87. Qu Q, Lee SJ and Boos W, J. Biol. Chem, 2004, 279, 47890-47897. [PubMed: 15364950]

88. Kim H-M, Chang Y-K, Ryu S-I, Moon S-G and Lee S-B, J. Mol. Catal. B-Enzym, 2007, 49, 98 103.

89. Woo EJ, Ryu SI, Song HN, Jung TY, Yeon SM, Lee HA, Park BC, Park KH and Lee SB, J. Mol. Biol, 2010, 404, 247-259. [PubMed: 20888836]

90. Ryu S-I, Kim J-E, Huong NT, Woo E-J, Moon S-K and Lee S-B, Enzyme Microb. Technol, 2010, 47, 249-256.

91. Kouril T, Zaparty M, Marrero J, Brinkmann H and Siebers B, Arch. Microbiol, 2008, 190, 355369. [PubMed: 18483808]

92. Urbanek BL, Wing DC, Haislop KS, Hamel CJ, Kalscheuer R, Woodruff PJ and Swarts BM, ChemBioChem, 2014, 15, 2066-2070. [PubMed: 25139066]

93. Meints LM, Poston AW, Piligian BF, Olson CD, Badger KS, Woodruff PJ and Swarts BM, J. Vis. Exp, 2017, e54485.

94. Rundell SR, Wagar ZL, Meints LM, Olson CD, O’Neill MK, Piligian BF, Poston AW, Hood RJ, Woodruff PJ and Swarts BM, Org. Biomol. Chem, 2016, 14, 8598-8609. [PubMed: 27560008]

95. Groenevelt JM, Meints LM, Stothard AI, Poston AW, Fiolek TJ, Finocchietti DH, Mulholand VM, Woodruff PJ and Swarts BM, J. Org. Chem, 2018, 83, 8662-8667. [PubMed: 29973045]

96. Siegrist MS, Swarts BM, Fox DM, Lim SA and Bertozzi CR, FEMS Microbiol. Rev, 2015, 39, 184-202. [PubMed: 25725012]

97. Kamariza M, Shieh P and Bertozzi CR, Methods Enzymol, 2018, 598, 355-369. [PubMed: 29306442]

98. Foley HN, Stewart JA, Kavunja HW, Rundell SR and Swarts BM, Angew. Chem. Int. Edit, 2016, 55, 2053-2057.

99. Rodriguez-Rivera FP, Zhou X, Theriot JA and Bertozzi CR, J. Am. Chem. Soc, 2017, 139, 34883495. [PubMed: 28075574]

100. Rodriguez-Rivera FP, Xiaoxue Z, Theriot JA and Bertozzi CR, Angew. Chem. Int. Edit, 2018, 57, 5267-5272.

101. Zhou X, Rodriguez-Rivera FP, Lim HC, Bell JC, Bernhardt TG, Bertozzi CR and Theriot JA, Nat. Chem. Biol, 2019, 15, 221-231. [PubMed: 30664686]

102. García-Heredia A, Pohane AA, Melzer ES, Carr CR, Fiolek TJ, Rundell SR, Lim HC, Wagner JC, Morita YS, Swarts BM and Siegrist MS, eLife, 2018, DOI: 10.7554/eLife.37243.

103. Hodges HL, Brown RA, Crooks JA, Weibel DB and Kiessling LL, Proc. Natl. Acad. Sci. U. S. A, 2018, 115, 5271-5276. [PubMed: 29703753] 
104. Fiolek TJ, Banahene N, Kavunja HW, Holmes NJ, Rylski AK, Pohane AA, Siegrist MS and Swarts BM, ChemBioChem, 2019, 20, 1282-1291. [PubMed: 30589191]

105. Kavunja HW, Piligian BF, Fiolek TJ, Foley HN, Nathan TO and Swarts BM, Chem. Commun, 2016, 52, 13795-13798.

106. Holmes NJ, Kavunja HW, Yang Y, Vannest BD, Ramsey CN, Gepford DM, Banahene N, Poston AW, Piligian BF, Ronning DR, Ojha AK and Swarts BM, ACS Omega, 2019, 4, 4348-4359. [PubMed: 30842987]

107. Kavunja HW, Biegas KJ, Banahene N, Stewart JA, Piligian BF, Groenevelt JM, Sein CE, Morita YS, Niederweis M, Siegrist MS and Swarts BM, J. Am. Chem. Soc, 2020, 142, 7725-7731. [PubMed: 32293873]

108. Sahile HA, Rens C, Shapira T, Andersen RJ and Av-Gay Y, ACS Omega, 2020, 5, 3661-3669. [PubMed: 32118181]

109. Kamariza M, Shieh P, Ealand CS, Peters JS, Chu B, Rodriguez-Rivera FP, Babu Sait MR, Treuren WV, Martinson N, Kalscheuer R, Kana BD and Bertozzi CR, Sci. Transl. Med, 2018, 10, eaam6310. [PubMed: 29491187]

110. Kamariza M, Keyser SGL, Utz A, Knapp BD, Ahn G, Cambier CJ, Chen T, Huang KC and Bertozzi CR, bioRxiv, 2020, DOI: 10.1101/2020.05.29.124008.

111. Peña-Zalbidea S, Huang AYT, Kavunja HW, Salinas B, Desco M, Drake C, Woodruff PJ, Vaquero JJ and Swarts BM, Carbohydr. Res, 2019, 472, 16-22. [PubMed: 30428395]

112. Cambier CJ, Banik SM, Buonomo JA and Bertozzi CR, bioRxiv, 2020, DOI: 10.1101/845081, 845081 .

113. Parker HL, Tomás RMF, Furze CM, Guy CS and Fullam E, Org. Biomol. Chem, 2020, 18, 36073612. [PubMed: 32350493]

114. Jain SK, Mol. Imaging Biol, 2017, 19, 341-347. [PubMed: 28155078]

115. Johnson DH, Via LE, Kim P, Laddy D, Lau C-Y, Weinstein EA and Jain S, Nucl. Med. Biol, 2014, 41, 777-784. [PubMed: 25195017]

116. Ankrah AO, van der Werf TS, de Vries EFJ, Dierckx RAJO, Sathekge MM and Glaudemans AWJM, Clin. Transl. Imaging, 2016, 4, 131-144. [PubMed: 27077068]

117. Banister S, Roeda D, Dolle F and Kassiou M, Curr. Radiopharm, 2010, 3, 68-80.

118. Hadfield AF, Hough L and Richardson AC, Carbohydr. Res, 1978, 63, 51-60.

119. Hadfield AF, Hough L and Richardson AC, Carbohydr. Res, 1979, 71, 95-102.

120. Basu S and Alavi A, J. Nucl. Med, 2008, 49, 17N-37N.

121. Tewson TJ, Welch MJ and Raichle ME, J. Nucl. Med, 1978, 19, 1339-1345. [PubMed: 104019]

122. Neal TR, Schumann WC, Berridge MS and Landau BR, J. Label. Compd. Radiopharm, 2005, 48, $845-854$.

123. Ghione M and Sanfilippo A, Giorn. Microbiol, 1957, 3, 189-196.

124. Arcamone F and Bizioli F, Gazz. Chim. Ital, 1957, 87, 896-902.

125. Umezawa S, Tatsuta K and Muto R, J. Antibiot. Ser. A, 1967, 20, 388-389.

126. Rose JD, Maddry JA, Comber RN, Suling WJ, Wilson LN and Reynolds RC, Carbohydr. Res, 2002, 337, 105-120. [PubMed: 11814442]

127. Wang J, Elchert B, Hui Y, Takemoto JY, Bensaci M, Wennergren J, Chang H, Rai R and Chang CW, Bioorg. Med. Chem, 2004, 12, 6397-6413. [PubMed: 15556758]

128. Sanki AK, Boucau J, Umesiri FE, Ronning DR, Sucheck SJ. Mol. BioSyst, 2009, 5, 945-956. [PubMed: 19668859]

129. Yang Y, Kulka K, Montelaro RC, Reinhart TA, Sissons J, Aderem A, and Ojha AK. Mol. Microbiol, 2011, 76, 13236-13239.

130. Islam MS, Richards JP and Ojha AK, Expert Rev. Anti. Infect. Ther, 2012, 10, 1055-1066. [PubMed: 23106280]

131. Ojha AK, Baughn AD, Sambandan D, Hsu T, Trivelli X, Guerardel Y, Alahari A, Kremer L, Jacobs WR and Hatfull GF, Mol. Microbiol, 2008, 69, 164-174. [PubMed: 18466296]

132. Yang Y, Bhati A, Ke D, Gonzalez-Juarrero M, Lenaerts A, Kremer L, Guerardel Y, Zhang P and Ojha AK, J. Biol. Chem, 2012, 288, 382-392. [PubMed: 23155047] 
133. Wolber JM, Urbanek BL, Meints LM, Piligian BF, Lopez-Casillas IC, Zochowski KM, Woodruff PJ and Swarts BM, Carbohydr. Res, 2017, 450, 60-66. [PubMed: 28917089]

134. Lee JJ, Lee S-K, Song N, Nathan TO, Swarts BM, Eum SY, Ehrt S, Cho S-N and Eoh H, Nat. Commun, 2019, 2928-2928. [PubMed: 31266959]

135. Magill SS, Edwards JR, Bamberg W, Beldavs ZG, Dumyati G, Kainer MA, Lynfield R, Maloney M, McAllister-Hollod L, Nadle J, Ray SM, Thompson DL, Wilson LE and Fridkin SK, N. Engl. J. Med, 2014, 370, 1198-1208. [PubMed: 24670166]

136. Danielson ND, Collins J, Stothard AI, Dong Q, Kalera K, Woodruff PJ, DeBosch BJ, Britton RA and Swarts BM, Chem. Commun, 2019, 55, 5009-5012.

137. Yuasa H, Izumi M and Hashimoto H, Curr. Top. Med. Chem, 2009, 9, 76-86. [PubMed: 19199997]

138. Shukla E, Thorat LJ, Nath BB and Gaikwad SM, Glycobiology, 2015, 25, 357-367. [PubMed: 25429048]

139. Marten AD, Stothard AI, Kalera K, Swarts BM and Conway MJ, J. Med. Entomol, 2020, 57, 1096-1103. [PubMed: 31982917]

140. Higgins CB, Zhang Y, Mayer AL, Fujiwara H, Stothard AI, Graham MJ, Swarts BM and DeBosch BJ, JCI Insight, 2018, 3. DOI: 10.1172/jci.insight.120794

141. Zhang Y, Higgins CB, Fortune HM, Chen P, Stothard AI, Mayer AL, Swarts BM and DeBosch BJ, Nat. Commun, 2019, 10, 1587-1587. [PubMed: 30962478]

142. Zhang Y and DeBosch BJ, Gut Microbes, 2020, 11, 1475-1482. [PubMed: 32329657]

143. Woudenberg-van Oosterom M, van Rantwijk F and Sheldon RA, Biotechnol. Bioeng, 1996, 49, 328-333. [PubMed: 18623584]

144. Park O-J, Kim D-Y and Dordick JS, Biotechnol. Bioeng, 2000, 70, 208-216. [PubMed: 10972932]

145. Vanaporn M and Titball RW, Virulence, 2020, 11, 1192-1202. [PubMed: 32862781] 


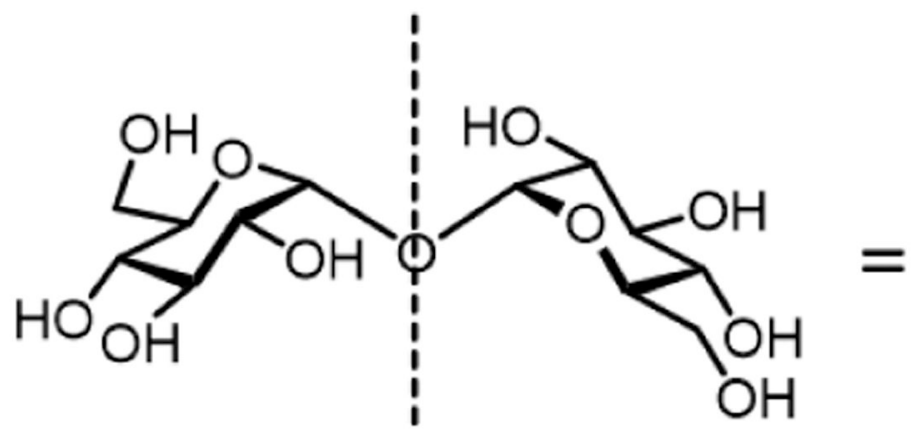

$\mathrm{C}_{2}$

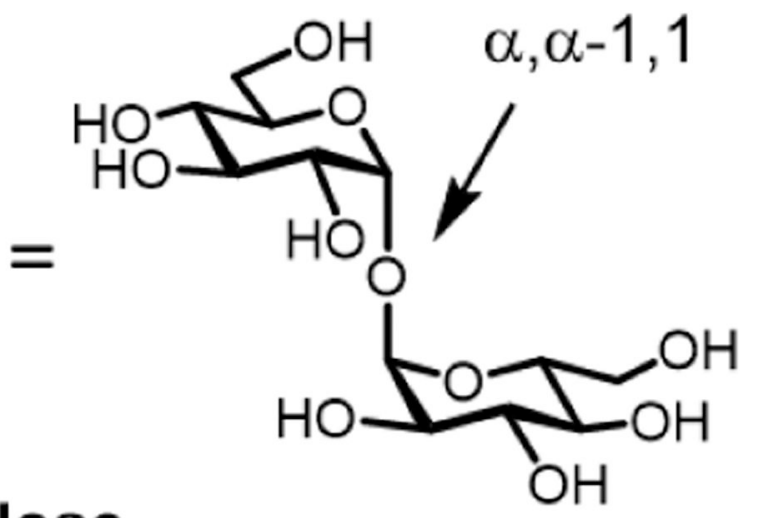

Trehalose

Figure 1.

Structure of trehalose. 
A ом [

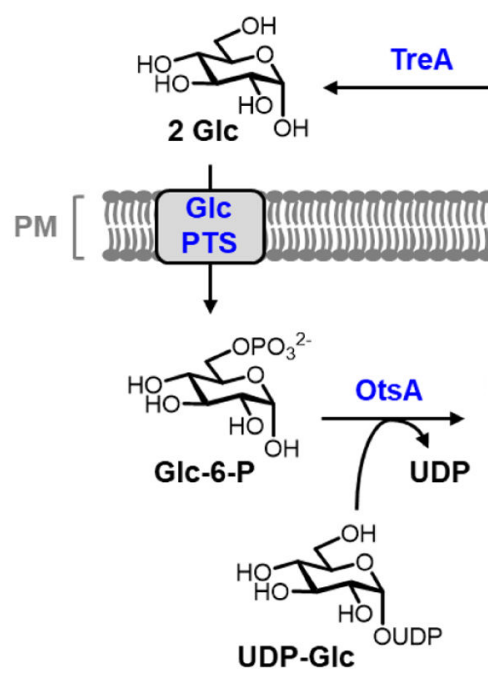

Trehalose

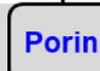

Trehalose
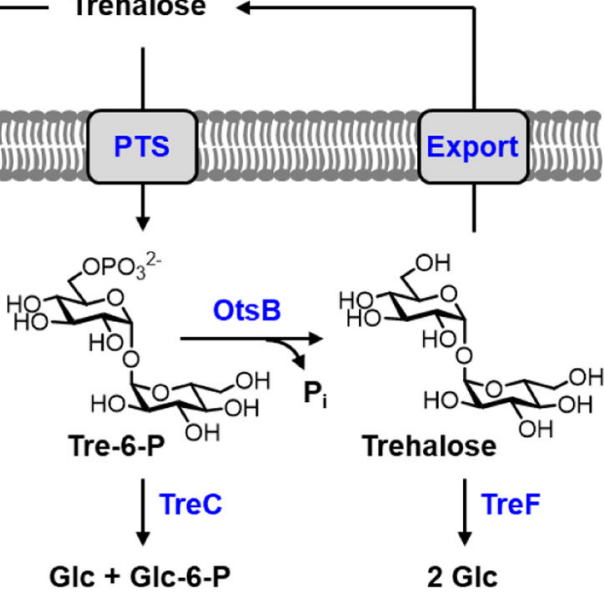

B
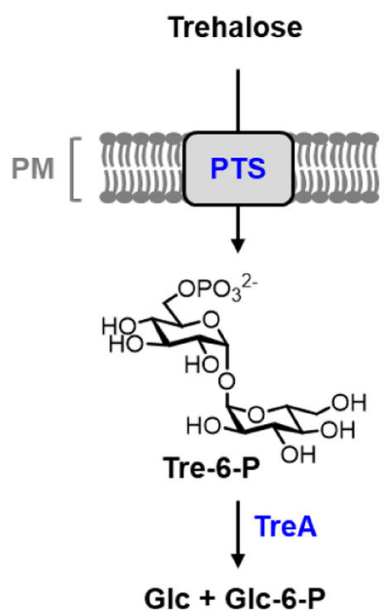

Figure 2.

(A) Representative trehalose metabolic pathways (A) in Gram-negative E. coli and (B) shared by Gram-positive $B$. subtilis and $C$. difficile. Similar pathways are found in many other bacteria. OM, outer membrane; PM, plasma membrane. 
Trehalose

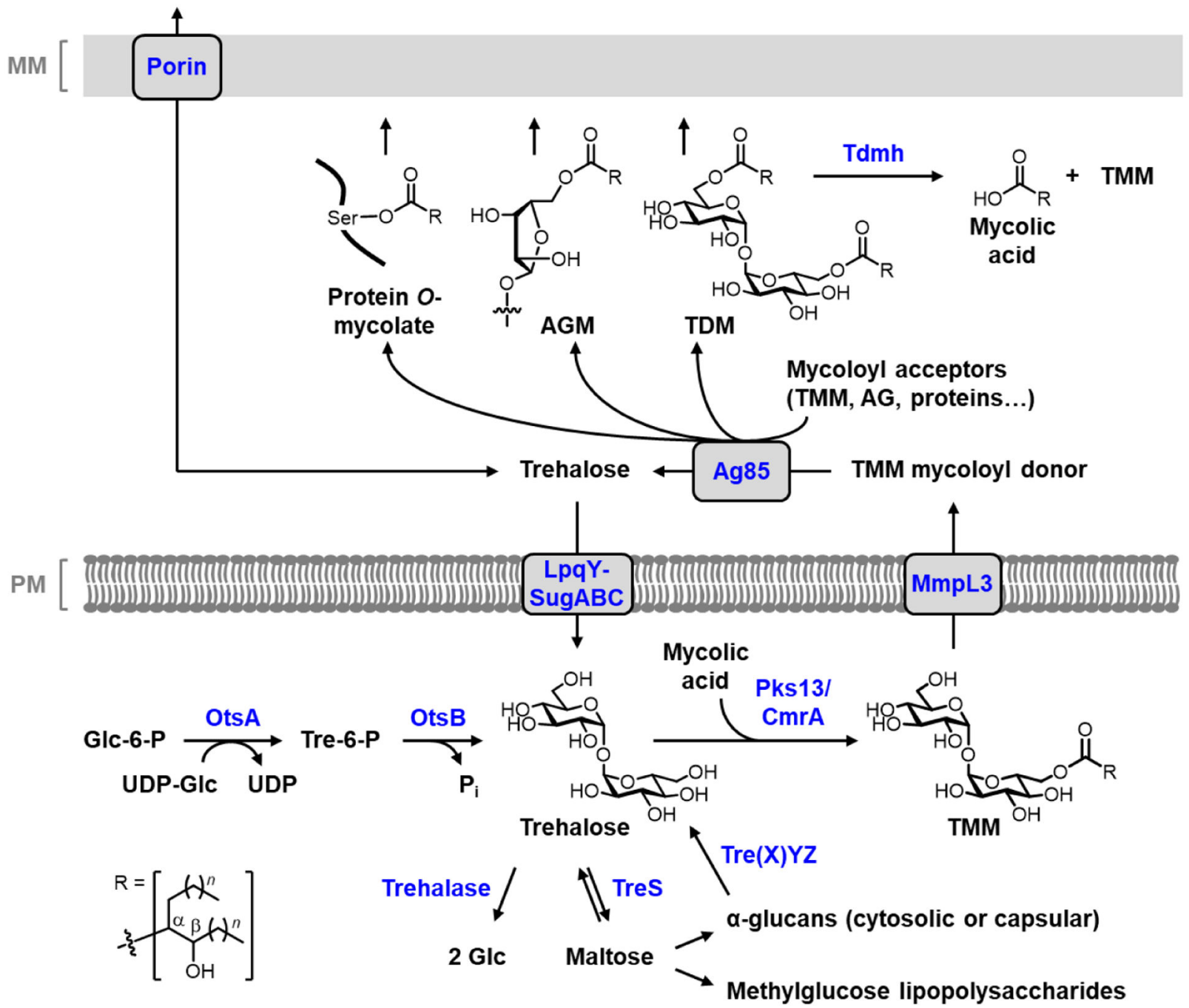

Figure 3.

(A) Trehalose metabolism in mycobacteria. MM, mycomembrane; PM, plasma membrane. 

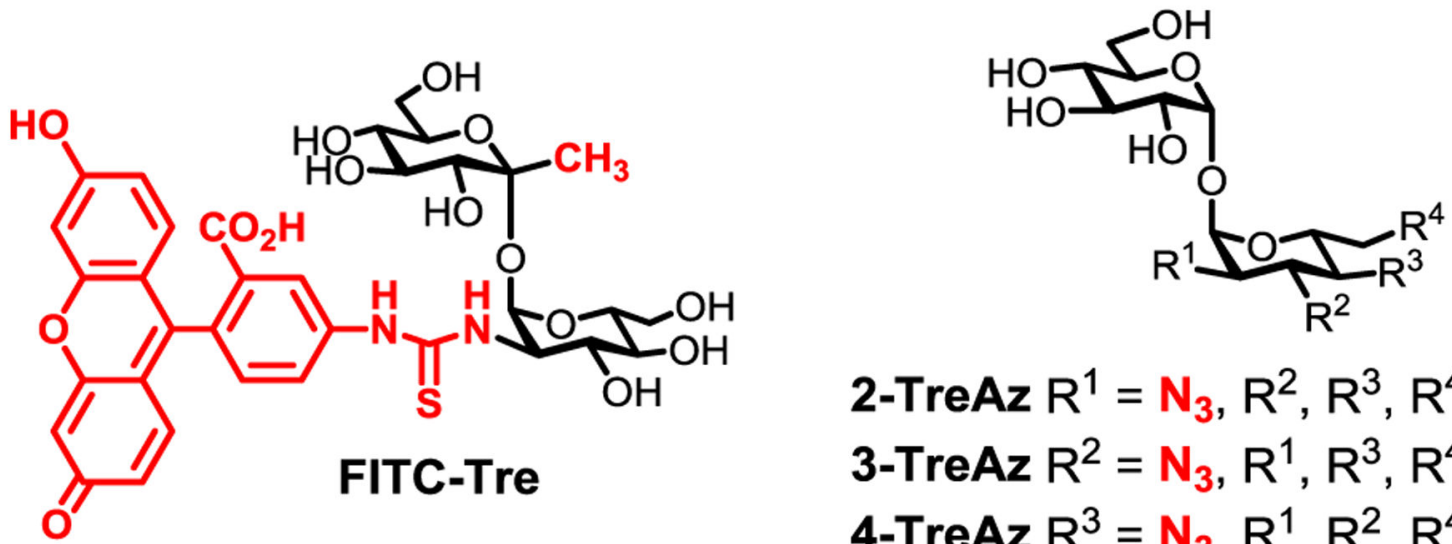

2-TreAz $\mathrm{R}^{1}=\mathrm{N}_{3}, \mathrm{R}^{2}, \mathrm{R}^{3}, \mathrm{R}^{4}=\mathrm{OH}$ 3-TreAz $\mathrm{R}^{2}=\mathrm{N}_{3}, \mathrm{R}^{1}, \mathrm{R}^{3}, \mathrm{R}^{4}=\mathrm{OH}$ 4-TreAz $\mathrm{R}^{3}=\mathrm{N}_{3}, \mathrm{R}^{1}, \mathrm{R}^{2}, \mathrm{R}^{4}=\mathrm{OH}$ 6-TreAz $\mathrm{R}^{4}=\mathrm{N}_{3}, \mathrm{R}^{1}, \mathrm{R}^{2}, \mathrm{R}^{3}=\mathrm{OH}$

Figure 4.

Examples of fluorescein- and azide-modified trehalose analogues used in mycobacteria research. 


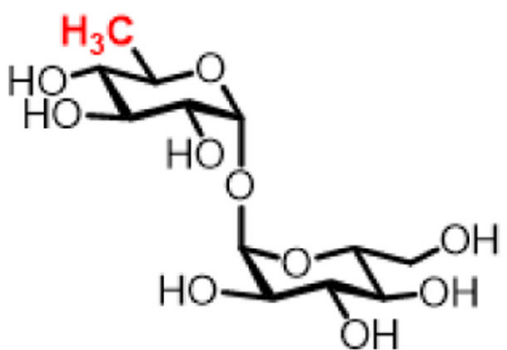

6-DeoxyTre E. gracilis TreP

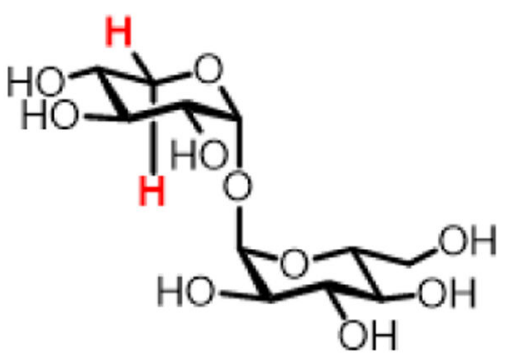

XyloTre

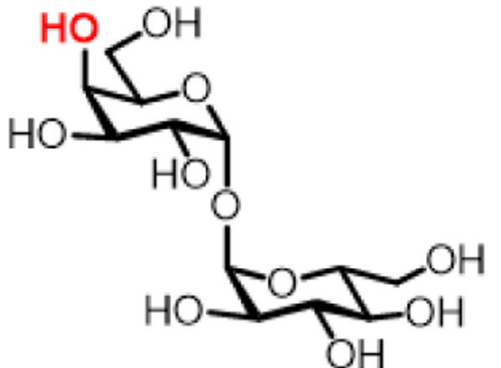

LactoTre

E. gracilis TreP

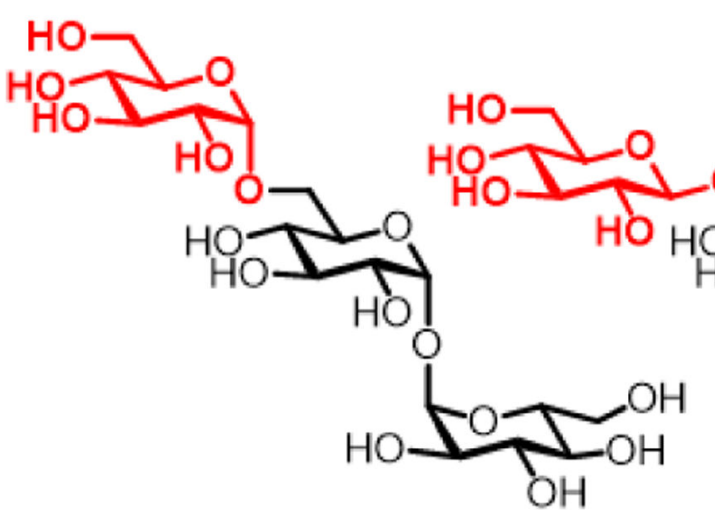

6-O- $\alpha$-GlcTre

T. brockii TreP
6-O- $\beta$-GIcTre

T. brockii TreP
T. brockii TreP

Figure 5.

Trehalose analogues synthesized via inverting TreP catalysis on at least a semi-preparative scale that enabled NMR confirmation of product structure. 


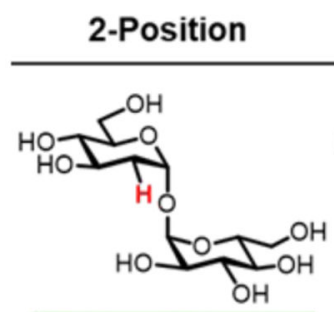

2-Deoxy Tre (>99\%)
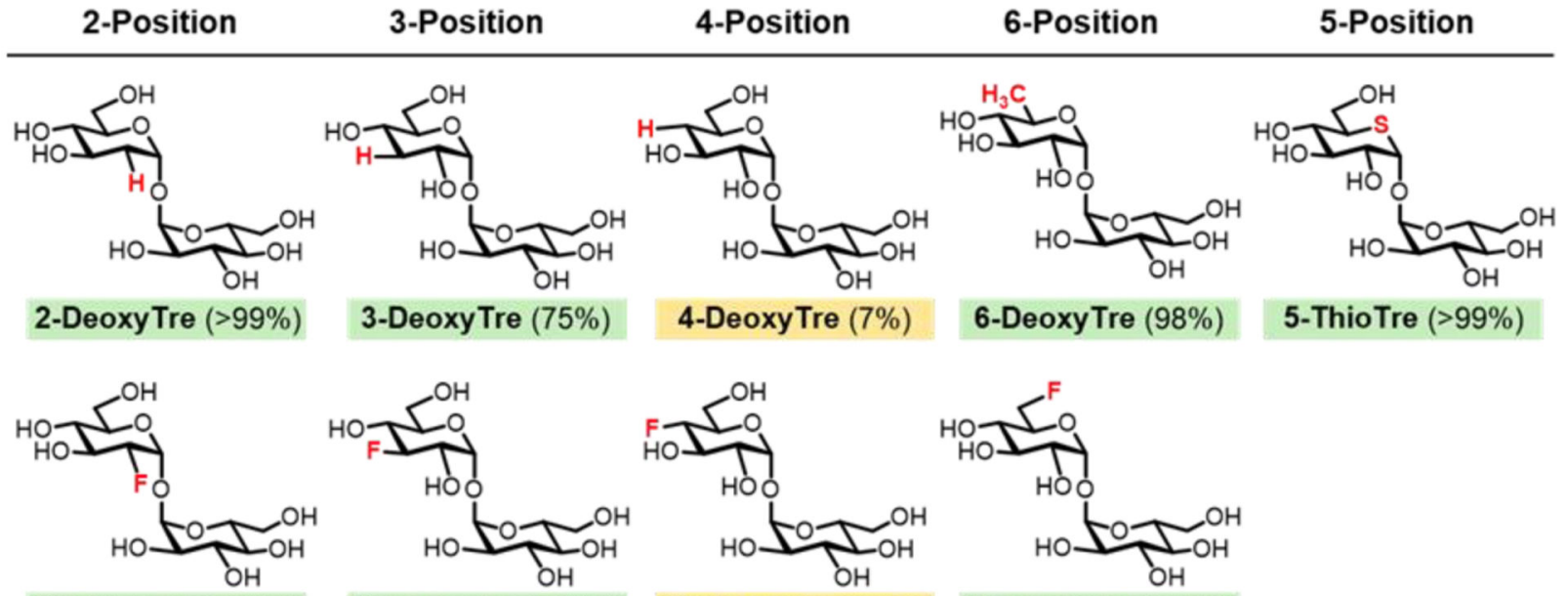

${ }^{19}$ F-2-FDTre (>99\%)

${ }^{19} \mathrm{~F}-3$-FDTre $(>99 \%)$

${ }^{19}$ F-4-FDTre (26\%)

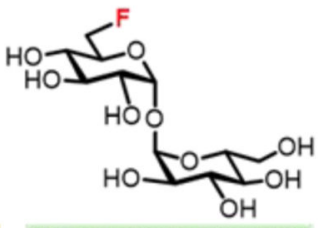

5-ThioTre (>99\%)
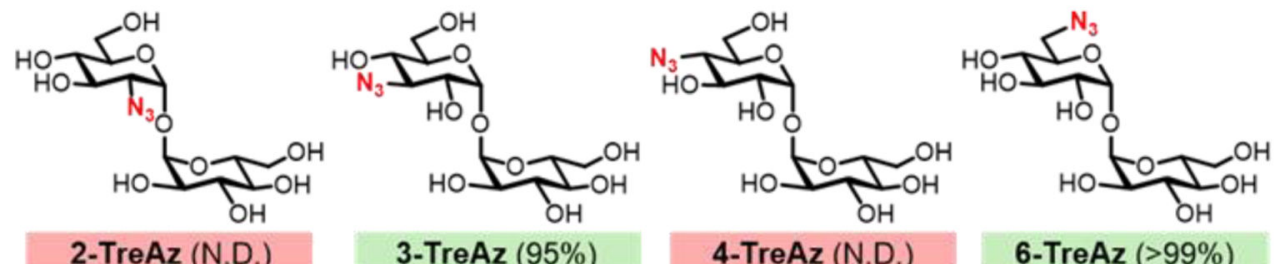

2-TreAz (N.D.)

4-TreAz (N.D.)

6-TreAz (>99\%)
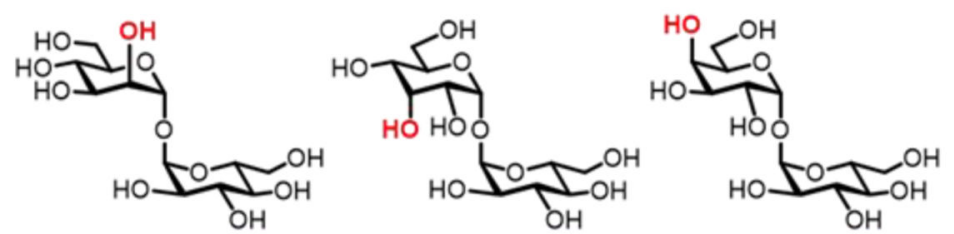

MannoTre (87\%)

AlloTre (N.D.)

LactoTre (N.D.)

Figure 6.

Trehalose analogues produced during initial development of the T. tenax TreT chemoenzymatic synthesis method (Scheme 5B). Percent conversions were determined by HPLC. Green, nearly complete or complete conversion; yellow, low or moderate conversion; red/N.D., product not detected. 


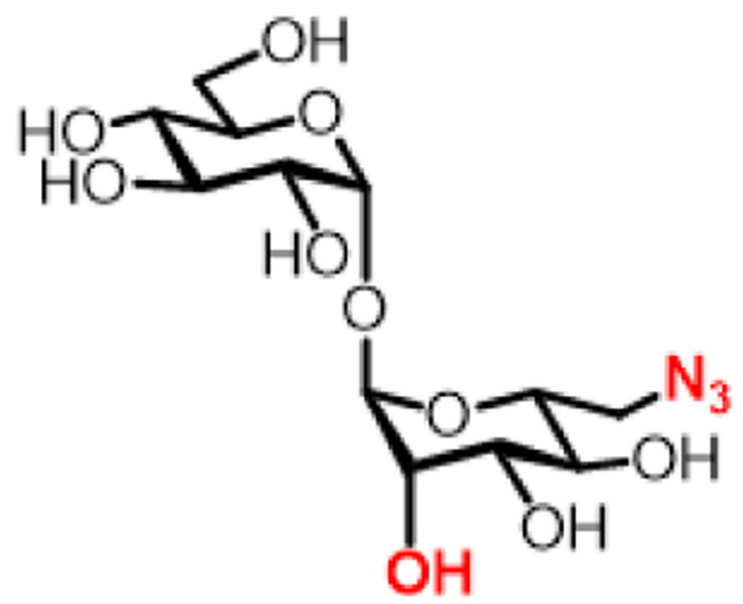

\section{6-MannoTreAz TreT}

Figure 7.

Epimeric TreAz analogues accessed by TreT catalysis.
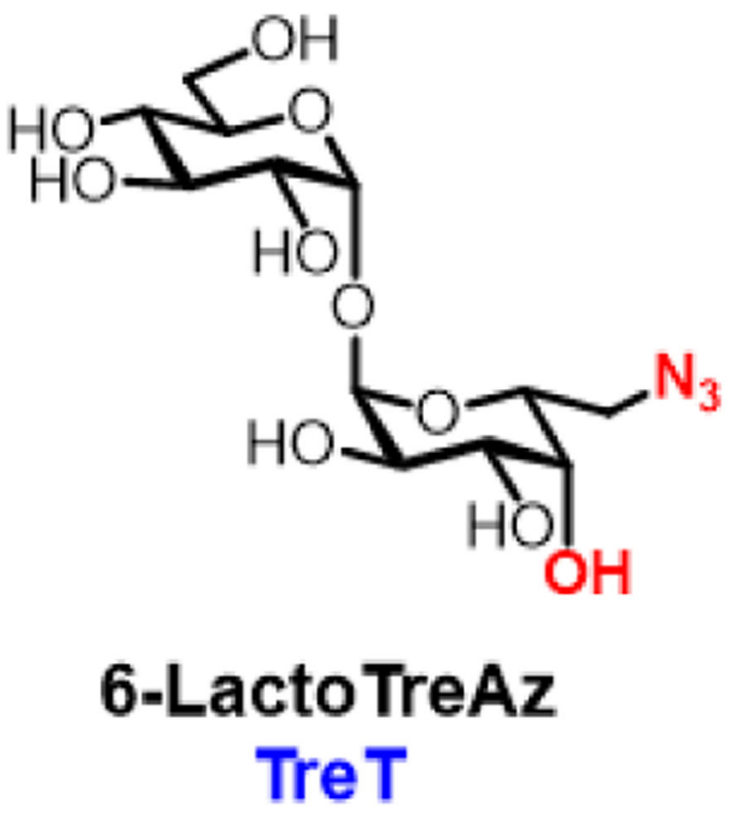


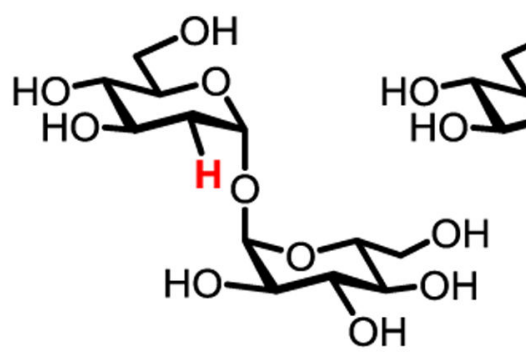

\section{2-DeoxyTre} TreT

\section{${ }^{19} \mathrm{~F}-2-F D T r e$} TreT<smiles>OCCC(O)C(O)O</smiles>

$\mathrm{HO}$<smiles>[Te]=[Po]</smiles><smiles>OC1OCC2OC(C2O)C(O)C(O)C1O</smiles><smiles>OC[C@H](O)[C@H](O)[C@H](O)[C@H](O)[C@H](O)CO</smiles>

\section{2-TreAz} Chemical synth.

MannoTre TreT<smiles>CC1OC(C)C(C)C(O)C(C)C1C</smiles><smiles>O</smiles><smiles>CCCCO</smiles>

$\mathrm{H}$

\section{6-DeoxyTre}

TreT
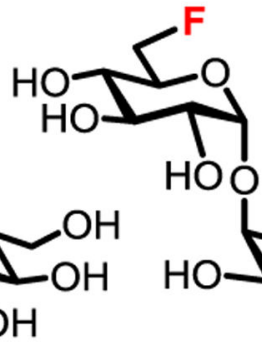

$\mathrm{HOO}$<smiles></smiles>

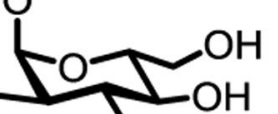

$\mathrm{OH}$

\section{${ }^{19}$ F-6-FDTre}

TreT

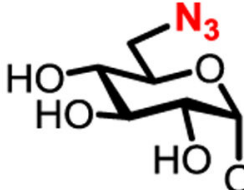<smiles>OCC1OC2OC(C1O)C(CO)C(O)C2O</smiles>

6-TreAz

TreT<smiles>OCC1C(O)C(O)C(O)C(O)C1O</smiles><smiles>CC1OC2OC1C(O)C(O)C(CO)C(O)C2O</smiles>

5-ThioTre TreT

Figure 8.

Panel of trehalose analogues evaluated for biofilm inhibition in M. smegmatis. 


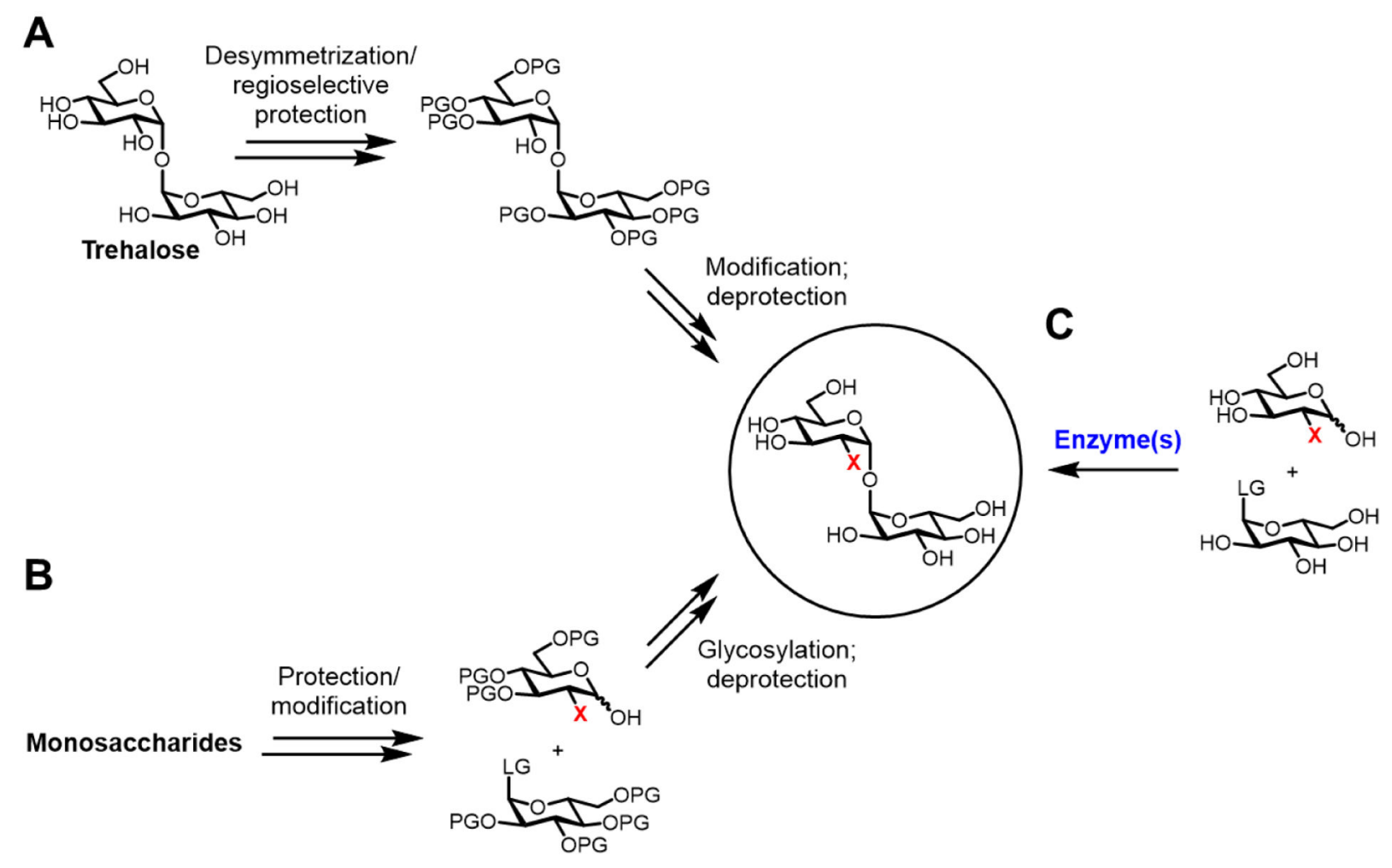

Scheme 1.

(A and B) Chemical and (C) chemoenzymatic approaches to trehalose analogue synthesis. $\mathrm{LG}=$ leaving group, $\mathrm{PG}=$ protecting group, $\mathrm{X}=$ modification . 


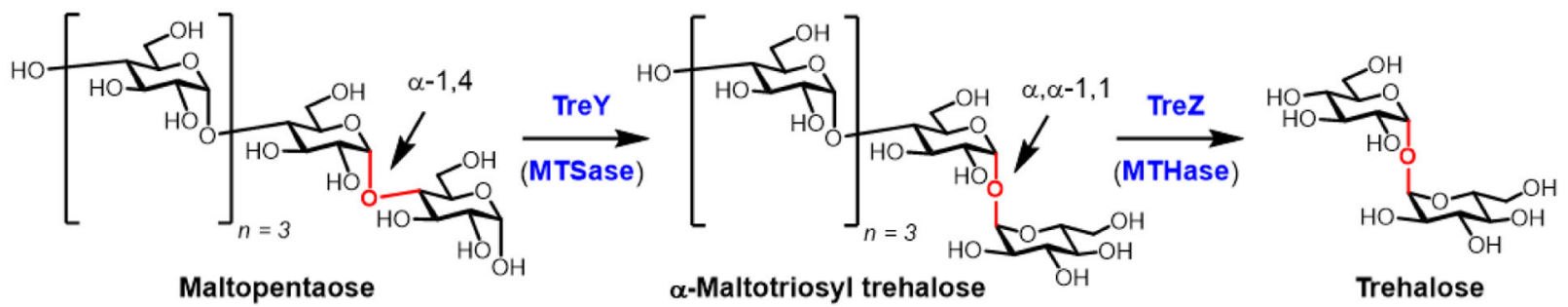

Scheme 2.

Industrial production of native trehalose from maltopentaose via the TreYZ (MTSase/ MTHase) pathway. 


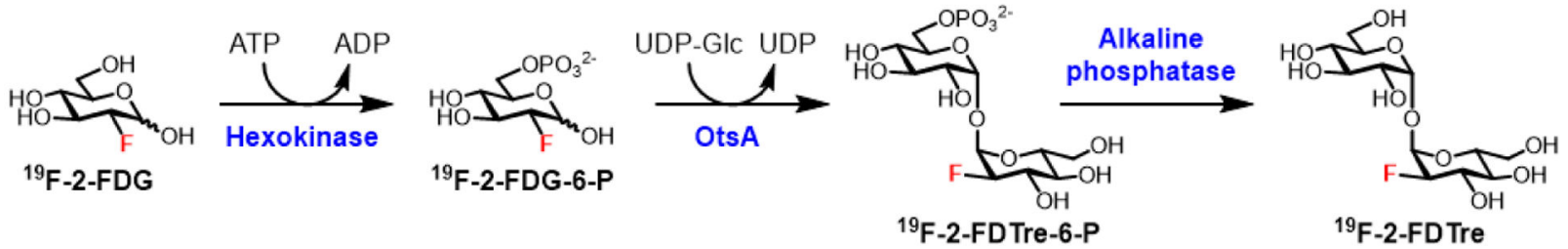

Scheme 3.

OtsAB (TPS/TPP)-inspired chemoenzymatic synthesis of ${ }^{19} \mathrm{~F}-2$-FDTre. 


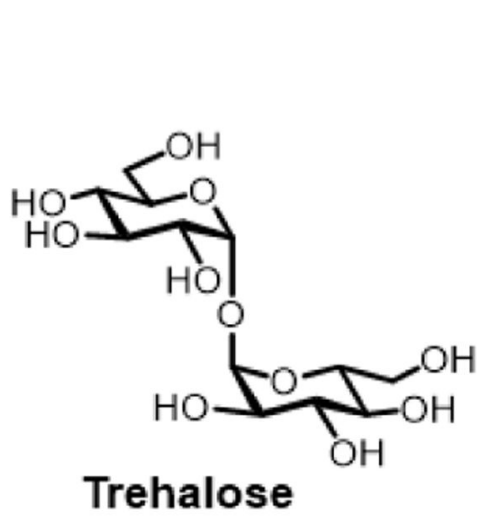

Trehalose
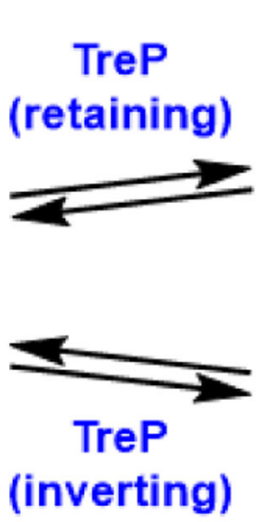
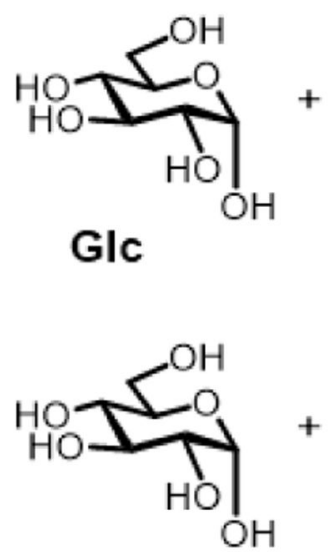

Glc

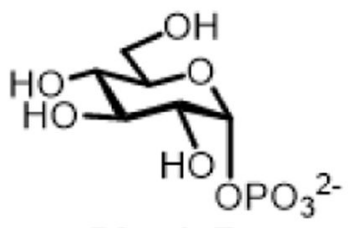

$\alpha$-Glc-1-P

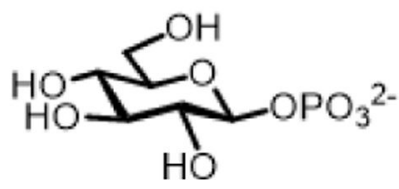

B-Glc-1-P

Scheme 4.

TreP enzymes catalyze the reversible conversion of trehalose to glucose and Glc-1-P. 


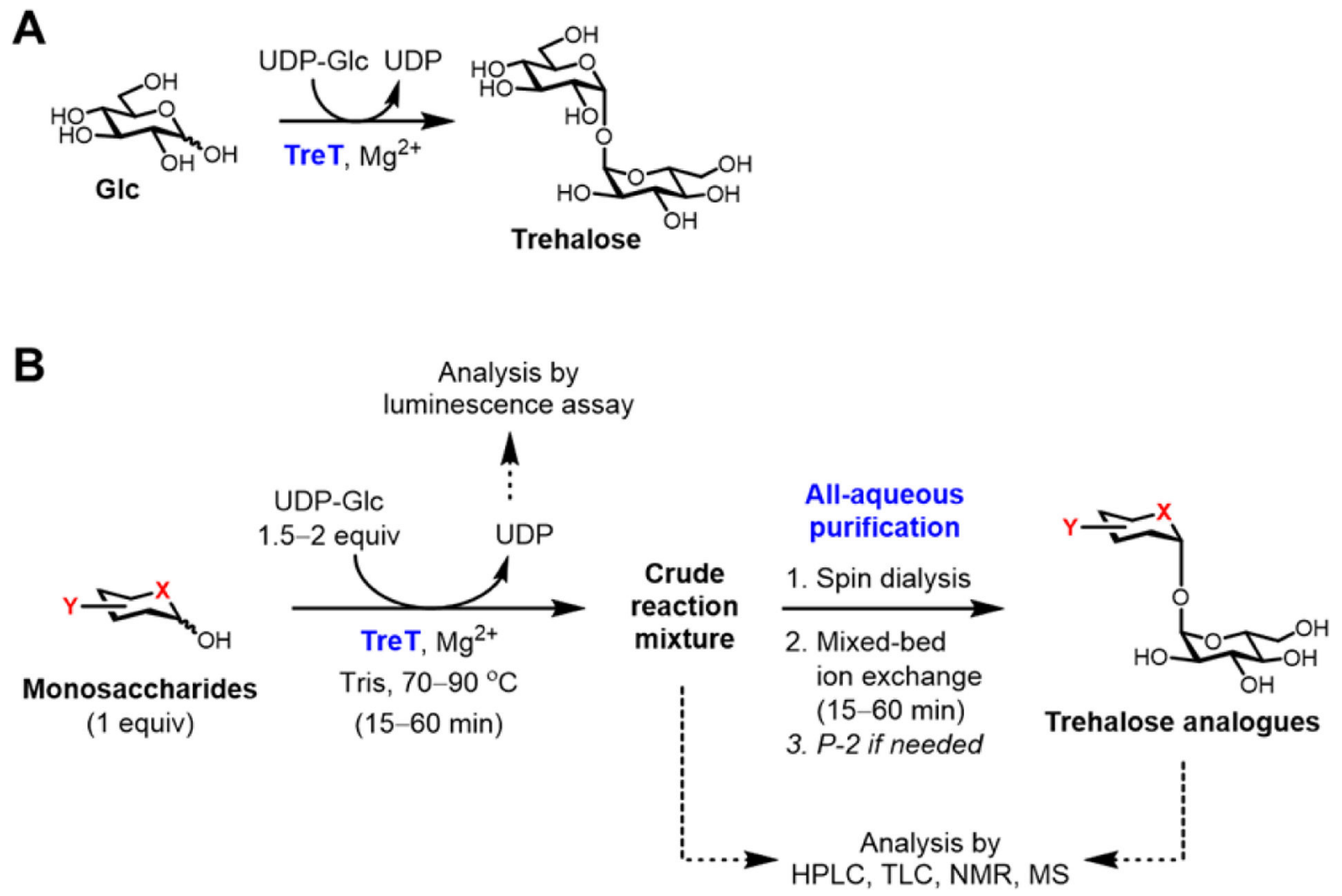

Scheme 5.

(A) Thermostable TreT enzyme catalyzes the conversion of glucose and UDP-Glc to trehalose. (B) In the standard acceptor variance mode, the T. tenax TreT chemoenzymatic synthesis method produces trehalose analogues from various monosaccharide acceptors and UDP-Glc. Optimized reaction and purification conditions and types of product analysis are indicated. See Figure 6 for analogues produced during initial reaction development. 


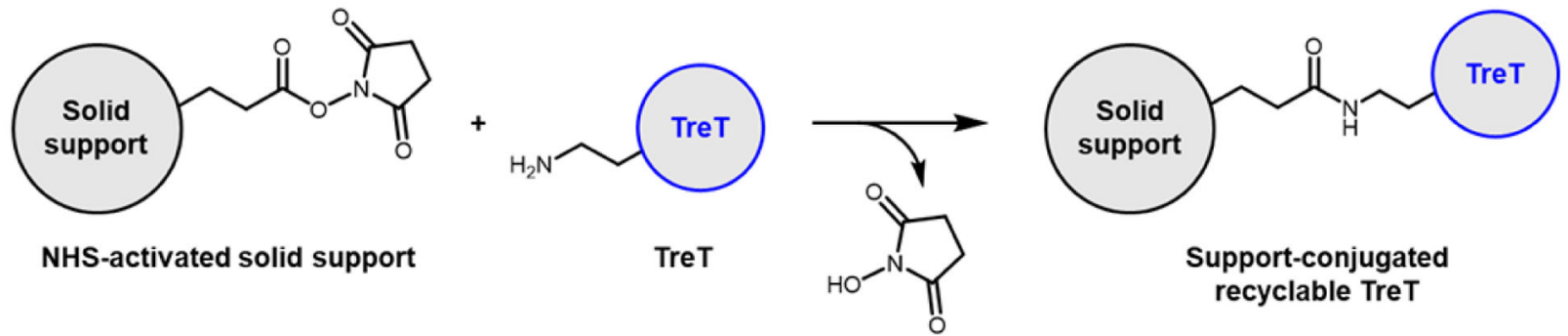

Scheme 6.

Preparation of immobilized TreT on magnetic and agarose solid supports. 


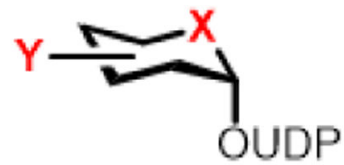

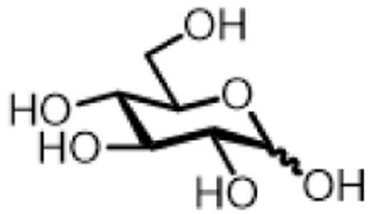

GIc

(1 equiv)
UDP-sugar

1.5-2 equiv

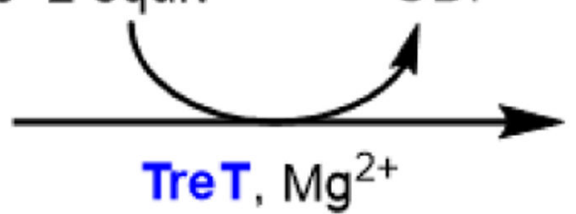

Tris, $70-90^{\circ} \mathrm{C}$

(15-60 $\mathrm{min}$ )

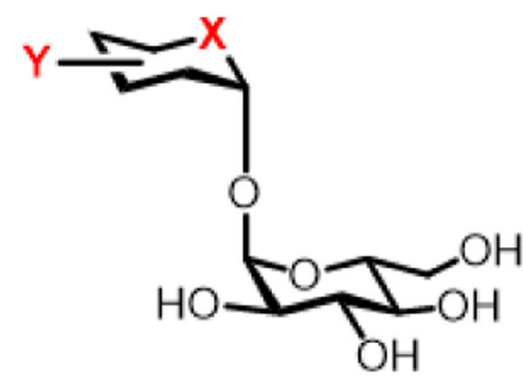

Trehalose analogues

Scheme 7.

In the donor variance mode, the $T$. tenax TreT chemoenzymatic synthesis method produces trehalose analogues from varied UDP-sugars and native glucose. 
A
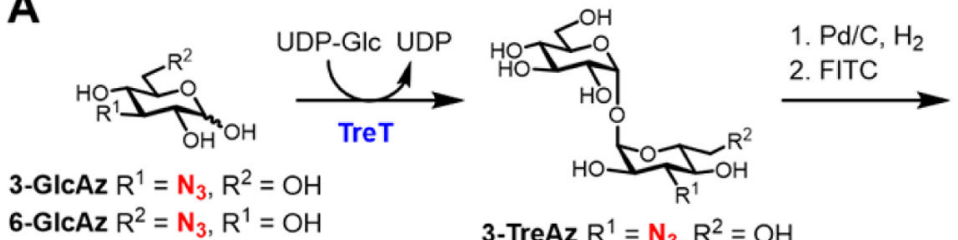

3-TreAz $R^{1}=\mathrm{N}_{3}, \mathrm{R}^{2}=\mathrm{OH}$

6-TreAz $R^{2}=N_{3}, R^{1}=O H$

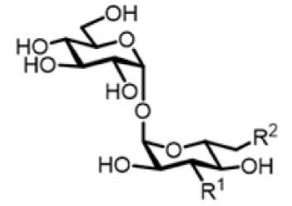

3-FITre $\mathrm{R}^{1}=\mathrm{FI}, \mathrm{R}^{2}=\mathrm{OH}$

6-FITre $\mathrm{R}^{2}=\mathrm{FI}, \mathrm{R}^{1}=\mathrm{OH}$

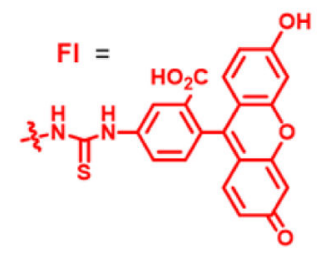

B
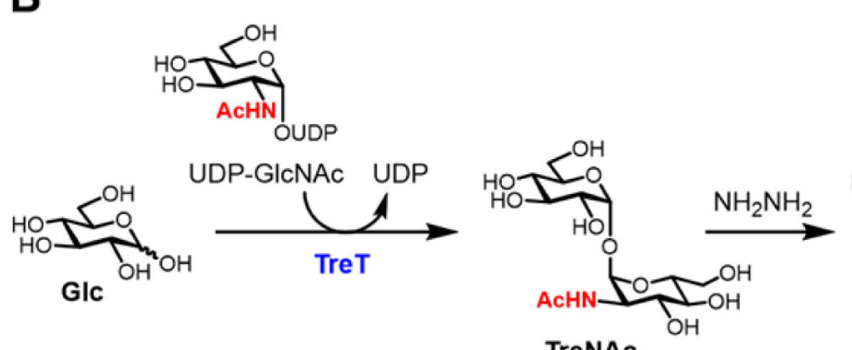

TreNAc

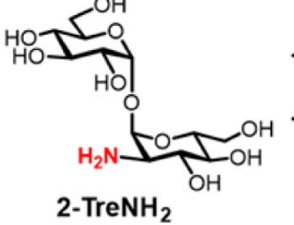

FITC

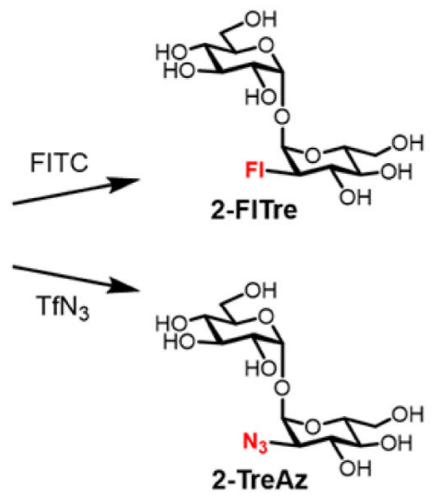

2- $\mathrm{TreNH}_{2}$

Scheme 8.

T. tenax TreT-based synthesis of fluorescent and clickable trehalose analogues using (A) the acceptor variance mode for 3- and 6-position-modified analogues and (B) the donor variance mode for 2-position-modified analogues. 


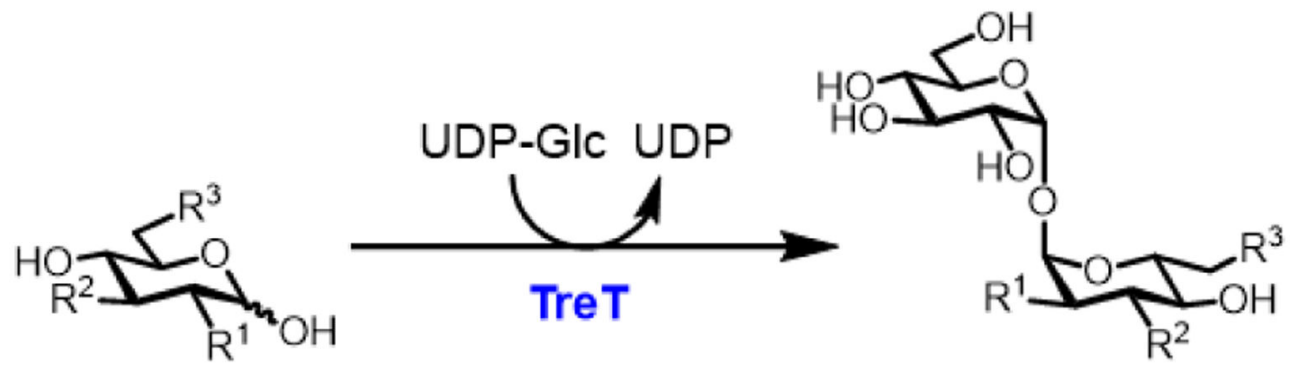

Non-radioactive substrates

${ }^{19} \mathrm{~F}-2$-FDG $\mathrm{R}^{1}={ }^{19} \mathrm{~F}, \mathrm{R}^{2}=\mathrm{R}^{3}=\mathrm{OH}$ ${ }^{19} \mathrm{~F}$-3-FDG $\mathrm{R}^{2}={ }^{19} \mathrm{~F}, \mathrm{R}^{1}=\mathrm{R}^{3}=\mathrm{OH}$ ${ }^{19} \mathrm{~F}-6$-FDG $\mathrm{R}^{3}={ }^{19} \mathrm{~F}, \mathrm{R}^{1}=\mathrm{R}^{2}=\mathrm{OH}$ Radioactive substrate ${ }^{18} \mathrm{~F}-2$-FDG $\mathrm{R}^{1}={ }^{18} \mathrm{~F}, \mathrm{R}^{2}=\mathrm{R}^{3}=\mathrm{OH}$
Non-radioactive products

${ }^{19} \mathrm{~F}$-2-FDTre $\mathrm{R}^{1}={ }^{19} \mathrm{~F}, \mathrm{R}^{2}=\mathrm{R}^{3}=\mathrm{OH}$ ${ }^{19} \mathrm{~F}$-3-FDTre $\mathrm{R}^{2}={ }^{19} \mathrm{~F}, \mathrm{R}^{1}=\mathrm{R}^{3}=\mathrm{OH}$ ${ }^{19} \mathrm{~F}-6$-FDTre $\mathrm{R}^{3}={ }^{19} \mathrm{~F}, \mathrm{R}^{1}=\mathrm{R}^{2}=\mathrm{OH}$ Radioactive product

${ }^{18} \mathrm{~F}$-2-FDTre $\mathrm{R}^{1}={ }^{18} \mathrm{~F}, \mathrm{R}^{2}=\mathrm{R}^{3}=\mathrm{OH}$

Scheme 9.

TreT-based synthesis of ${ }^{19} \mathrm{~F}$ - and ${ }^{18} \mathrm{~F}$-FDTre analogues as potential PET probes for in vivo imaging of mycobacteria and other trehalose-utilizing bacteria. 